[20] R. Hoffmann, P. Hofmann, J. Am. Chem. Soc. 98 (1976) 598.

[21] T. A. Albright, P. Hofmann, R. Hoffmann, J. Am. Chem. Soc. 99 (1977) 7546.

[22] R. Hoffmann, J. Chem. Phys. 39 (1963) 1397

[23] R. H. Summerville, R. Hoffmann, J. Am. Chem. Soc. 98 (1976) 7240; H. Berke, R. Hoffmann, ibid. 100 (1978) 7224; J. W. Richardson, W. C. Nieuwpoort, R. R. Powell, W. F. Edgell, J. Chem. Phys. 36 (1962) 1057; H. Basch, A. Viste, H. B. Gray, Theor. Chim. Acra 3 (1965) 458.

[24] R. Osman, P. Coffey, J. R. Van Wazer, Inorg. Chem. 15 (1976) 287.

[25] M. Di Vaira, L. Sacconi, P. Stoppioni, unveroffentlicht.

[26] K. Wade, Inorg. Nucl. Chem. Letr. 8 (1972) 559.

[27] A. S. Foust, M. S. Foster, L. F. Dahl, J. Am. Chem. Soc. 91 (1969) 5631.

[28] A. S. Foust, M. S. Foster, L. F. Dahl, J. Am. Chem. Soc. 91 (1969) 5633.

[29] R. H. Summerville, R. Hoffmann, J. Am. Chem. Soc. 101 (1978) 3821

[30] L. Sacconi, S. Midollini, M. Di Vaira, unveröffentlicht.
[31] Siehe Fußnote 11 in Lit. [33].

[32] H. H. Erdmann, M. von Unruch, Z. Anorg. Allg. Chem. 32 (1902) 439.

[33] C. Bianchini, M. Di Vaira, A. Meli, L. Sacconi, Inorg. Chem. 20 (1981) 1169

[34] C. Bianchini, M. Di Vaira, A. Meli, L. Sacconi, Angew. Chem. 92 (1980) 412; Angew. Chem. Int. Ed. Engl. 19 (1980) 405

[35] C. Bianchini, M. Di Vaira, A. Meli, L. Sacconi, J. Am. Chem. Soc. I03 (1981) 1448.

136] L. Fabrizzi, L. Sacconi, Inorg. Chim. Acra 36 (1979) L 407.

[37] G. Thiele, G. Zonbek, H. A. Lindner, J. Ellermann, Angew. Chem. 90 (1978) 133; Angew. Chem. Int. Ed. Engl. 17 (1978) 135.

[38] S. Midollini, A. Orlandini, L. Sacconi, Angew. Chem. 91 (1979) $93:$ An gew. Chem. Int. Ed. Engl. 18 (1979) 81

[39] C. Mealli, S. Midollini, S. Moneti, L. Sacconi, Crusr. Struct. Commun. 9 (1980) 1017

[40] F. Cecconi, C. A. Ghilardi, S. Midollini, A. Orlandini, J. Chem. Soc Chem. Commun. 1982, 229.

\title{
Anomalien des flüssigen Wassers
}

\author{
Von Elmar Wolfgang Lang und Hans-Dietrich Lüdemann*
}

Bei tiefen Temperaturen $(T<300 \mathrm{~K})$ zeigt flüssiges Wasser viele ungewöhnliche physikalisch-chemische Eigenschaften, die durch die starke Direktivität der Wasserstoffbrückenbindung und die damit verbundene energetische Bevorzugung einer tetraedrischen Nahordnung mit schlechter Raumausfüllung verursacht werden. Diese Anomalien werden im metastabilen Bereich unterhalb der Schmelzdruckkurve wesentlich ausgeprägter. Im vorliegenden Beitrag werden die Experimentalbefunde anhand der neueren Theorien diskutiert und mit den Ergebnissen von Computer-Simulationen am kalten Wasser verglichen. Die Anomalien des Wassers, die sich z. B. im Dichtemaximum der flüssigen Phase oder in der Abnahme der Viskosität mit dem Druck manifestieren, sind auf den Bereich $T<300 \mathrm{~K}$ und $p \leq 200 \mathrm{MPa}$ beschränkt. Bei diesen Drücken zeigt das unterkühlte Wasser mit fallender Temperatur ein ungewöhnliches Verhalten: Alle isobaren Eigenschaften deuten auf einen Phasenübergang bei $T \approx\left(T_{\mathrm{K}}-50 \mathrm{~K}\right)$, dessen physikalische Natur noch nicht eindeutig beschrieben werden kann. Dieses Phänomen ist bis jetzt ausschließlich im flüssigen Wasser beobachtet worden. - Künftig wird man unterkühlte wäßrige Lösungen für kinetische Untersuchungen verwenden und dadurch den zugänglichen dynamischen Bereich der Untersuchung wäßriger Lösungen erheblich erweitern können.

\section{Einleitung}

Unter den klimatischen Bedingungen unseres Planeten ist Wasser die bei weitem häufigste Flüssigkeit. Seine hohe spezifische Wärmekapazität und Schmelzwärme dämpfen die täglichen und jahreszeitlichen Temperaturschwankungen auf der Erdoberfläche und haben somit wesentlichen Einfluß auf das Klima. Die ungewöhnlichen physikalischen Eigenschaften des Wassers bei Temperaturen um $300 \mathrm{~K}$ und Atmosphärendruck waren eine der wichtigsten Voraussetzungen für die präbiologische und biologische Evolution auf der Oberfläche der Erde ${ }^{[1-4]}$.

Die Fähigkeit und ausgeprägte Tendenz des Wassermoleküls, durch seine beiden Protonen und die beiden freien Elektronenpaare an vier Wasserstoffbrücken sowohl als Donor als auch als Acceptor zu partizipieren, bewirkt in der reinen Flüssigkeit eine tetraedrische Nahordnung.

[*] Prof. Dr. H.-D. Lüdemann, Dr. E. W. Lang

Institut für Biophysik und Physikalische Biochemie der Universität

Postfach 397, D-8400 Regensburg 2
Diese Struktur ist letztlich Ursache für alle Anomalien des flüssigen Wassers und der Eismodifikationen. Die Anomalien manifestieren sich unter anderem auch in der großen Häufigkeit, mit der in Wasser irreguläre Lösungen apolarer Stoffe sowie Mikroheterogenitäten in der Verteilung gelöster Nichtelektrolyte vorkommen ${ }^{[5.6]}$.

Proteine und Nucleinsäuren bilden in wäßriger Lösung oft wohlgeordnete Strukturen wie Helices oder Faltblattanordnungen, für deren Entstehung neben den intramolekularen Wechselwirkungen auch die intermolekularen Beiträge, die vom Wasser herrühren, wichtig sind ${ }^{[7.8]}$.

Der Beitrag des Wassers zur Stabilisierung dieser Strukturen besteht zum einen in der Bildung intermolekularer Wasserstoffbrücken mit den polaren Gruppen der Biopolymere; zum anderen werden apolare Kohlenwasserstoffgruppen entweder durch die hydrophobe Wechselwirkung ins Innere der Polymere abgedrängt, oder das Wasser wird durch hydrophobe Hydratation an der Kontaktfläche zwischen apolarer Gruppe und Lösungsmittel strukturiert $^{[9.10]}$. 
Die Tendenz zur Bildung einer tetraedrischen Nahordnung und damit relativ offener Strukturen mit schlechter Raumausfüllung nimmt mit fallender Temperatur zu; diese Nahordnung bewirkt das Dichtemaximum, das man in leichtem Wasser bei $277 \mathrm{~K}$ und in Deuteriumoxid bei $285 \mathrm{~K}$ beobachtet. Die ungewöhnlichen Eigenschaften des Wassers sind bei Temperaturen unterhalb des Dichtemaximums wesentlich stärker ausgeprägt.

Leider liegt die Temperatur des Dichtemaximums nur wenig oberhalb der Schmelztemperatur, so daß im Gebiet thermodynamischer Stabilität nur ein kleiner Temperaturbereich der Untersuchung zugänglich ist. Befreit man Wasser durch gründliche Reinigung von allen keimbildenden Verunreinigungen, so gelingt es, die heterogene Nucleation zu vermeiden und unterkühltes flüssiges Wasser für lange Zeit im metastabilen Zustand zu halten.

Es ist leicht einsehbar, daß die Handhabung unterkühlten Wassers um so unproblematischer wird, je kleiner die Proben sind, da das abnehmende Volumen die Wahrscheinlichkeit für das Auftreten homogener Nucleation verringert. Die meisten der Untersuchungen im metastabilen Bereich wurden daher entweder mit Wasser vorgenommen, das in engen Kapillaren ${ }^{[1]}$ enthalten war, mit kleinen Tröpfchen, die als Nebel in staubfreier Luft ${ }^{[12]}$ zerstäubt waren, oder mit Wassertröpfchen, die in einem apolaren wasserunlöslichen Träger emulgiert waren ${ }^{[13]}$. Die unterkühlten Emulsionen werden durch einen wasserunlöslichen Emulgator stabilisiert und können monatelang gelagert werden. Bei Atmosphärendruck ist die emulgierte wäßrige Phase bis $233 \mathrm{~K}$ unterkühlbar ${ }^{[14 !}$. Die gleiche Temperatur $T_{\mathbf{H}}$ der homogenen Nucleation wird beobachtet, wenn man Wassertröpfchen in einem Temperaturgradienten absinken läßt ${ }^{[12]}$.

Wasser/Cycloalkan-Emulsionen eignen sich zur Untersuchung der Druckabhängigkeit von $T_{\mathrm{H}}$. Kanno, Speedy und Angell ${ }^{\left[15 .{ }^{16]}\right.}$ haben $T_{\mathrm{H}}$ für $\mathrm{H}_{2} \mathrm{O}$ und $\mathrm{D}_{2} \mathrm{O}$ bis zu Drükken von $300 \mathrm{MPa}$ durch Differentialthermoanalyse bestimmt. Fig. 1 zeigt, daß $T_{\mathrm{H}}$ noch wesentlich stärker mit

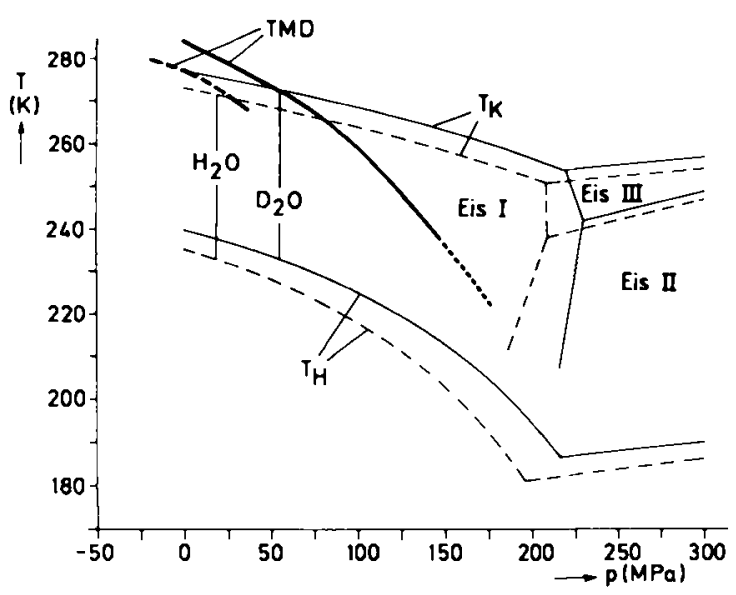

Fig. I. $p$ - $T$-Projektion des Phasendiagramms von $\mathrm{H}_{2} \mathrm{O}$ und $\mathrm{D}_{2} \mathrm{O}$. $T_{\mathrm{K}}$ Schmelztemperatur, $T_{11}$ : Temperatur der homogenen Nucleation [15, 16]. Eingetragen ist zusătzlich die Temperatur der maximalen Dichte (TMD) [8790] (siehe Abschnitt 3.1).

dem Druck sinkt als die Schmelztemperatur $T_{\mathrm{K}}$ und daß Wasser bei $200 \mathrm{MPa}$ bis $-92^{\circ} \mathrm{C}$ unterkühlbar ist. Der me- tastabile Temperaturbereich wird also bei hohem Druck wesentlich größer.

Im folgenden soll an einigen Beispielen gezeigt werden, daß die in diesem Bereich gefundenen Eigenschaften des metastabilen Wassers eine stetige Fortsetzung der im Stabilitätsbereich beobachteten Eigenschaften sind, und daß alle Anomalien des flüssigen Wassers im Unterkühlungsgebiet wesentlich ausgeprägter werden. Die physikalischen Eigenschaften unterkühlten Wassers werden von Angell in Band 7 der von Franks herausgegebenen Reihe: „Water A Comprehensive Treatise" umfassend dargestellt ${ }^{1811}$.

\section{Die Gleichgewichtsstruktur des flüssigen Wassers}

\subsection{Vorbemerkungen}

Die makroskopischen Eigenschaften einer Flüssigkeit und ihre Struktur lassen sich im Prinzip aus den physikalischen Eigenschaften ihrer Bestandteile und den Wechselwirkungen zwischen ihnen berechnen. Eine rigorose quantenmechanische Behandlung ist für ein System von $\approx 10^{23}$ Freiheitsgraden nicht sinnvoll und praktisch auch nicht möglich, da die Mikrozustände beliebig dicht beieinander liegen (Energieabstand $\Delta \approx \exp \left(-10^{23}\right) \mathrm{erg}$ ) und somit experimentell nicht entschieden werden kann, in welchem Mikrozustand sich das System befindet. Analog ist es in der klassischen Mechanik nicht möglich, die zur Lösung der Bewegungsgleichungen eines Systems von größenordnungsmäßig $10^{23}$ Teilchen nötigen Anfangsbedingungen zu bestimmen. Es sind deshalb über den Zustand solcher Systeme nur statistische Aussagen möglich. Die statistische Mechanik versucht, die makroskopischen Größen als über Verteilungsfunktionen gewogene Mittelwerte mit analytischen Methoden darzustellen. Die Anwendung bekannter Integralgleichungen ${ }^{[17]}$ der statistischen Mechanik auf flüssiges Wasser ist jedoch bisher wenig erfolgreich gewesen $^{[18-20]}$. Computer-Simulationen ${ }^{[21]}$ dagegen führen die Mittelwertbildung numerisch an wegen der erforderlichen Rechenzeit sehr kleinen Systemen von $\leqslant 10^{3}$ Teilchen aus. Dabei wurden zwei Rechenverfahren angewendet: Mit der molekulardynamischen Methode löst man die klassischen Newton-Euler-Bewegungsgleichungen und bildet zeitliche Mittelwerte; mit der Monte-Carlo-Methode errechnet man Ensemblemittelwerte. Da bei Systemen von maximal $10^{3}$ Teilchen Randeffekte eine bedeutende Rolle spielen, verwenden beide Methoden entweder periodische Randbedingungen und einen Abschneideradius, bis zu dem die Wechselwirkungen der Moleküle berücksichtigt werden, oder die Ewald-Summation ${ }^{[22.23]}$. Neben den im folgenden diskutierten Simulationen wurden auch Modelle mit Erfolg angewendet, die flüssiges Wasser über chemische Gleichgewichte zwischen zwei oder mehreren möglichen Zuständen der freien oder gebundenen Hydroxygruppen beschreiben $^{[24,25]}$.

\subsection{Modellpotentiale und Computer-Simulationen}

In molekulardynamischen Rechnungen und in MonteCarlo-Rechnungen benötigt man das Gesetz für die zwi- 
schenmolekularen Kräfte in der flüssigen Phase. Dieses Problem kann bei einfachen Flüssigkeiten in guter Näherung als Zweikörperproblem behandelt werden. Im flüssigen Wasser dagegen sind, wie quantenmechanische Rechnungen gezeigt haben ${ }^{[26-28]}$, Mehrkörperkräfte nicht vernachlässigbar. Da die genaue Form des Wechselwirkungspotentials in der flüssigen Phase nicht bekannt ist, werden in Computer-Simulationen Modellpotentiale ${ }^{[29-32]}$ verwendet.

a) Geeignet sind effektive Molekülpaarpotentiale ${ }^{|33-3.5|}$, die mit Punktladungsmodellen aus einfachen parametrisierten Funktionen aufgebaut sind. Die Parameter werden mit wenigen experimentellen Größen optimiert (z. B. BNS- ${ }^{[36-38]}$, ST2 $-^{[39-43]}$, Rowlinson- ${ }^{[44-46]}$, TIPSVerfahren $\left.{ }^{[47]}\right)$. Diese Modelle berücksichtigen innere Freiheitsgrade und Dissoziationseffekte nicht. Wird die elektrostatische Wechselwirkung zwischen Punktladungen durch eine elektrische Multipolentwicklung ersetzt, erhält man nicht-additive Potentiale, die Polarisationseffekte miteinbeziehen ${ }^{[32]}$.

b) Verwendet werden außerdem parametrisierte Funktionen (Coulomb-Potentiale, Exponentialfunktionen), die numerisch an quantenmechanische ab-initio-Rechnungen des Dimerpotentials angepaßt werden (z. B. MCYVerfahren $^{[48-51]}{ }^{[31.52 .53]}$. Diese Modelle lassen den Einกuß von Mehrkörperkräften unberücksichtigt.

c) Mit empirischen Atompaarpotentialen zwischen H- und O-Atomen der Ladung $\pm \eta \cdot e$ (z. B. CF-Verfahren ${ }^{|54-56|}$, $\left.{ }^{[57-59]}\right)$ können auch innere Freiheitsgrade und Dissoziationseffekte beschrieben werden. Hinzufügen einer nicht-additiven Wechselwirkung zwischen Ladungswolken, deren Form in Analogie zur klassischen Elektrostatik gewählt wird, führt zu Modellpotentialen, die auch Polarisationseffekte erfassen ${ }^{[60-66]}$.

Zur Beschreibung der Gleichgewichtsstruktur einer molekularen Flüssigkeit dienen Atompaarkorrelationsfunktionen $g_{\alpha \beta}(R)^{[29]}$. Sie können sowohl mit Streuexperimenten als auch mit Computer-Simulationen bestimmt werden. Fig. 2 zeigt $g_{\mathrm{oo}}(R)$ für die kondensierten Phasen von $\mathrm{H}_{2} \mathrm{O}$. Den Atompaarkorrelationsfunktionen ist zu entnehmen, $\operatorname{da} \beta$

a) Lage und Anzahl nächster Nachbarn in flüssigem Wasser denen in der kristallinen Phase ähneln (tetraedrische Nahordnung). Die Lage der übernächsten Nachbarn ist durch eine breite Verteilung von $\mathrm{O}-\mathrm{O}-\mathrm{O}$-Bindungswinkeln mit einem Schwerpunkt beim Tetraederwinkel gekennzeichnet. Für Abstände $R \geq 8 \AA$ ist keine Lagekorrelation mehr zu erkennen;

b) die Maxima in $g_{\mathrm{OH}}(R)$ und $g_{\mathrm{HH}}(R)$ bei Abständen auftreten, wie sie für nahezu lineare $H$-Brücken zu erwarten sind (ausführliche Diskussion siehe ${ }^{[29-32]}$ ).

Unter hohem hydrostatischen Druck ${ }^{[43.67]}$ erinnert die Funktion $g_{o o}(R)$ an Verteilungsfunktionen einfacher Flüssigkeiten.

Die Veränderungen in den Funktionen $g_{\alpha \beta}(R)$ mit steigender Dichte zeigen an, daß sich die $\mathrm{H}$-Brücken verbiegen und einander durchdringende $\mathrm{H}$-Brückennetzwerke entstehen. Diese Befunde lassen erkennen, da $B$ die Gleichgewichtsstruktur flüssigen Wassers am besten als ein ungeordnetes Netzwerk mehr oder weniger stark gestörter Was-
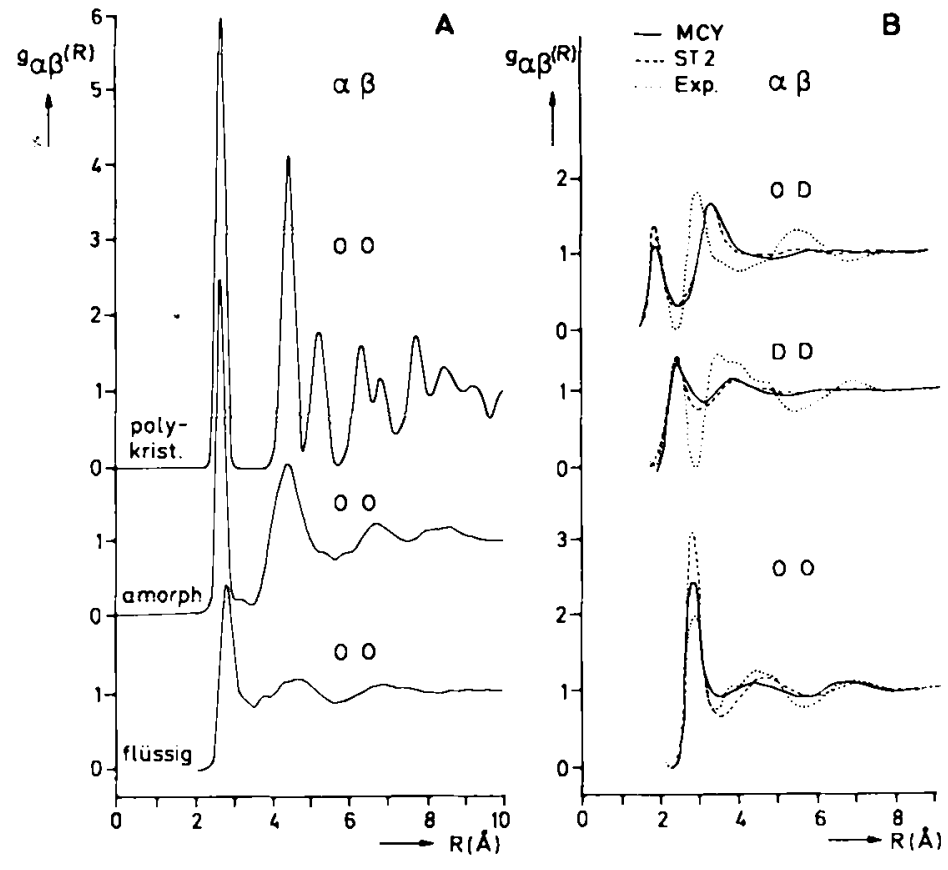

Fig. 2. Atompaarkorrelationsfunktionen $g_{n \mid ;}(R)$ im flüssigen Wasser. A) Vergleich der durch Röntgen-Streuung ermittelten $g_{\text {oo }}(R)$ für polykristallines, amorphes und flüssiges Wasser $(300 \mathrm{~K})$ [74]; B) Vergleich der berechneten und aus experimentellen Ergebnissen gemittelten $g_{\text {(t) }}(R)$ [182, 183] (MCY [48-51], ST2 [39-43], siehe Text).

serstoffbrücken anzusehen ist (zur Topologie siehe [39.53.68.69]). Teilt man die Moleküle entsprechend der Zahl der H-Brücken ein, an denen sie beteiligt sind, so zeigen alle Rechnungen eine unimodale Verteilung. Untersuchungen der Polygonverteilung ergeben, daß ungeradzahlige und geradzahlige Ringe mit vergleichbarer Häufigkeit auftreten und daß Ringe mit bis zu 11 Molekülen vorkommen. Eine Analyse der Charakteristika von $\mathrm{H}$-Brücken führt zu Verteilungsfunktionen der Bindungswinkel mit einem Maximum bei nicht-linearen $\mathrm{H}$-Brücken ${ }^{[33.69]}$. Der Vernetzungsgrad einer vorgegebenen Konfiguration von Wassermolekülen sowie die Struktur und Verteilung von Clustern mit $n$ Wassermolekülen wurden ebenfalls untersucht ${ }^{[7]}$. Die statistischen Kenngrößen des $\mathrm{H}$-Brückennetzwerks wurden in Abhängigkeit von der mittleren $Z$ ahl von $H$ Brücken pro Molekül ( $\overline{\mathrm{n}})$ bestimmt. Das H-Brückennetzwerk hat bei der Dichte $\rho=1 \mathrm{~g} / \mathrm{cm}^{3}$ und Raumtemperatur eine Bindungsperkolationsschwelle bei $\bar{n} \approx 1.3^{\left[{ }^{*}\right]}$. Alle $\mathrm{Ab}$ schätzungen von $\bar{n}$ für diese Bedingungen liegen über diesem Wert; demnach ist Wasser zu jedem Zeitpunkt ein raumausfüllendes Netzwerk von $\mathrm{H}$-brückengebundenen Molekülen. Der mittlere Anteil ringbildender H-Brücken ist erst für $\bar{n}>1.3$ von Null verschieden. Ringstrukturen sind somit ein wesentliches Merkmal des unregelmäßigen H-Brückennetzwerks im flüssigen Wasser ${ }^{[69]}$.

Alle bisherigen Betrachtungen hatten die in ComputerSimulationen erzeugte momentane Struktur des Systems (I-Struktur ${ }^{[71]}$ ) zum Gegenstand. Hirala et al. ${ }^{[72]}$ und Belch et al. ${ }^{[7]}$ haben durch Mittelung der Schwerpunkts- und Winkelkoordinaten über eine Zeitspanne von $0.2 \mathrm{ps}$, die etwa der Periodendauer von behinderten Translationen und Librationen entspricht, eine Struktur erzeugt, die der

[*] Unter Bindungsperkolationsschwelle versteht man das erstmalige Auftreten eines die gesamte Probe durchziehenden H-Brückennetzwerks. 
von Eisenberg und Kauzmann ${ }^{[71]}$ diskutierten V-Struktur sehr nahe kommt. Diese unterscheidet sich von der IStruktur durch

a) eine Paarkorrelationsfunktion $g_{\text {oo }}(R)$ mit einem schärferen und höheren ersten Maximum,

b) eine stärkere Bevorzugung nahezu linearer $\mathrm{H}$-Brücken,

c) eine starke Zunahme der $\mathbf{H}$-Brückenbindungen,

d) eine starke Zunahme der Zahl nicht quervernetzter Polygone unterschiedlicher Größe $(n=3-11)$.

Diese Ergebnisse hängen nicht wesentlich von der Mittelungsperiode ab. Die Stabilisierung des ungeordneten Netzwerks in der V-Struktur ist dem Entzug der mittleren thermischen Anregungsenergie $k_{\mathrm{B}} T$ aus der I-Struktur äquivalent. Die behinderten Translationen und Librationen können deshalb als thermische Anregungen des ungeordneten $\mathrm{H}$-Brückennetzwerks verstanden werden.

\subsection{Das ,random network“-Modell ${ }^{[74]}$}

Hauptresultat der Computer-Simulationen flüssigen Wassers ist die Erkenntnis, daß das wesentliche Strukturmerkmal der Flüssigkeit ein ungeordnetes Netzwerk von Molekülen ist, die durch gerichtete $\mathrm{H}$-Brücken variabler Bindungsenergie und -geometrie wechselwirken. Das Netzwerk ordnet sich auf der Zeitskala von Diffusionsprozessen kontinuierlich um und erscheint für höherfrequente Schwingungen quasistatistisch. Dieses Bild ist Grundlage des "random network“-Modells ${ }^{[75]}$, das mit experimentellen Daten der kristallinen, der amorphen festen und der flüssigen Phase des Wassers entwickelt wurde ${ }^{[76]}$.

Die quasistatische Struktur des ungeordneten Netzwerks wird neben dem mittleren Abstand nächster Nachbarn $R_{\text {OO }}$ vornehmlich durch zwei Verteilungen beschrieben: die Streuung der Abstände nächster Nachbarn $\Delta R_{\text {OO }}^{2}$ und die Abweichungen von einer linearen $\mathrm{H}$-Brükkengeometrie $\theta_{\mathrm{H}}^{2}\left(\theta_{\mathbf{H}}=\mathrm{H}\right.$-Brückenbindungswinkel). Behinderte Translationen und Librationen werden als thermische Anregungen dieses quasistatischen Netzwerks aufgefaßt.

Neben der strukturellen Charakterisierung des ungeordneten Netzwerks haben Sceats und Rice ${ }^{[77]}$ auch ein Wechselwirkungspotential der Wassermoleküle innerhalb des „random network"-Modells entwickelt. Da wiederum sehr stark auf spektroskopische Daten Bezug genommen wird, ist das Potential nur in einem kleinen Bereich des Konfigurationsraumes gültig. Dies ist jedoch der wichtige Bereich nahe dem Energieminimum, in welchem $\mathbf{H}$-BrückenWechselwirkungen die gegenseitige Lage der Moleküle entscheidend bestimmen. Das ,random network“-Potential wird als Potenzreihe in den Abweichungen von einer Referenzkonfiguration geschrieben. Dabei werden nur zwei Koordinaten verwendet, nämlich $R_{\text {oo }}$ und $\theta_{\mathrm{H}}$.

Das effektive „random network“-Potential wird zusammen mit dem Modell zur Berechnung thermodynamischer Potentiale sowie statischer Antwortfunktionen $n^{(*)}$ benützt. Die Übereinstimmung ist im allgemeinen sehr befriedigend $^{[73.78-80]}$.

[*] Statische Antwortfunktionen (response functions) sind z. B. der thermische Ausdehnungskoeffizient $\alpha$, die isotherme Kompressibilität $\kappa$, oder die Wärmekapazität bei konstantem Druck $C_{1}$.

\section{Statische Eigenschaften des unterkühlten Wassers}

Im folgenden wird an einigen thermodynamischen Größen des unterkühlten Wassers gezeigt, daß sich die Eigenschaften der unterkühlten Phase stetig an diejenigen der stabilen flüssigen Phase anschließen. Es wird versucht, die Sonderstellung des flüssigen Wassers an ausgewählten Beispielen zu veranschaulichen. Eine vollständigere und umfangreichere Darstellung gibt Angel/ ${ }^{[81]}$, dessen Gruppe in den letzten Jahren viele der hier beschriebenen Resultate erarbeitet hat.

\subsection{Die Dichte $p$}

Bei $0.1 \mathrm{MPa}$ ist die Dichte für $\mathrm{H}_{2} \mathrm{O}$ bis $239 \mathrm{~K}$ und für $\mathrm{D}_{2} \mathrm{O}$ bis $244 \mathrm{~K}$ in Kapillaren von ca. $10 \mu \mathrm{m}$ Durchmesser gemessen worden ${ }^{[82]}$. Die so ermittelten Dichten stimmen mit den in Emulsionen gemessenen ${ }^{[14]}$ überein. Diese Daten sind zusammen mit den Dichten im Stabilitätsbereich $^{[\times 3]}$ in Fig. 3 als reduzierte Größen $\rho_{\mathrm{r}}, T_{\mathrm{r}}$ aufgetragen

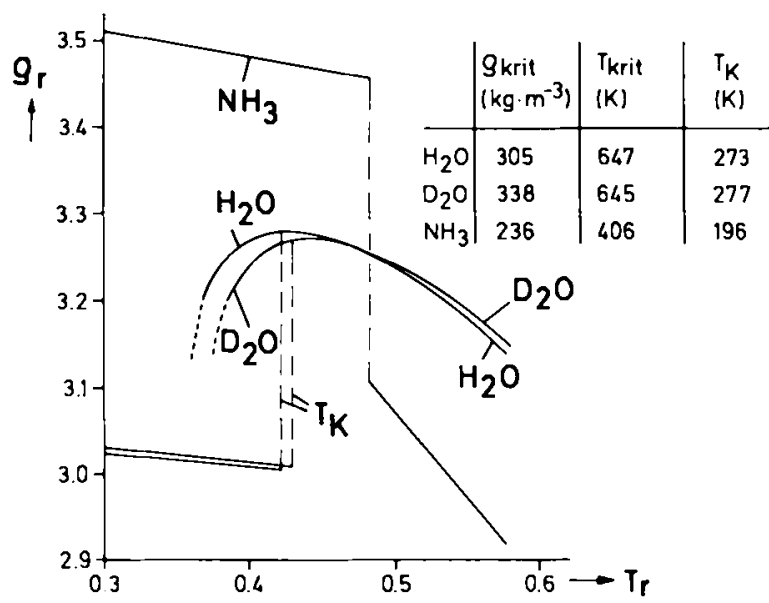

Fig. 3. $\rho_{1}-T_{1}$-Diagramm von $\mathrm{H}_{2} \mathrm{O}$ und $\mathrm{D}, \mathrm{O}$ bei $0.1 \mathrm{MPa}$ und von $\mathrm{NH}_{3}$ beim Săttigungsdampfdruck. $T_{\mathrm{K}}$ : Schmelztemperatur.

$\rho_{1} \equiv \rho_{\mathrm{r}, \mathrm{r}} \cdot \rho_{\mathrm{k} \text { III }}^{-1} ; T_{\mathrm{r}} \equiv T \cdot T_{\mathrm{hril}}^{-1}$

In leichtem Wasser beobachtet man bei $T=277 \mathrm{~K}$ ein Dichtemaximum und eine geringfügige Dichteabnahme bei Temperaturerniedrigung bis zum Schmelzpunkt. Im Unterkühlungsbereich setzt sich diese Dichteabnahme stärker fort, so daß $\rho_{\text {flüssig }}(T)$ im Bereich von $220-240 \mathrm{~K}$ der Dichte von Eis Ih entsprechen würde ${ }^{l^{*}}$. Die Volumenausdehnung setzt in $\mathrm{D}_{2} \mathrm{O}$ wesentlich früher ein $\left(T_{p_{\text {man }}}=285 \mathrm{~K}\right)$. Unterhalb von $\approx 310 \mathrm{~K}$ ist die reduzierte Dichte von $\mathrm{D}_{2} \mathrm{O}$ kleiner als die reduzierte Dichte von $\mathrm{H}_{2} \mathrm{O}$. Fig. 3 zeigt auch die Temperaturabhängigkeit der Dichte von Ammoni$a^{[84.85]}$, die wie üblich mit fallender Temperatur steigt. Bei $\mathrm{NH}_{3}$ ist auch die Kristallisation mit einer Dichtezunahme verbunden.

Kanno und Angell ${ }^{[86]}$ haben im Unterkühlungsbereich die Dichte von $\mathrm{D}_{2} \mathrm{O}$ bis zu $243 \mathrm{~K}$ und $140 \mathrm{MPa}$ verfolgt. Fig. 1 zeigt die Druckabhängigkeit der Temperatur der maximalen Dichte (TMD) aus diesen Messungen, zusammen mit älteren Befunden ${ }^{[87-89]}$ an $\mathrm{H}_{2} \mathrm{O}$, die Henderson und

[*] Eis Ih ist die bei niedrigen Drücken thermodynamisch stabile hexagonale Eismodifikation. 
Speed $y^{[90]}$ kürzlich bis zu negativen Drücken von -16 $\mathrm{MPa}$ ausdehnten. Die Extrapolation der für $\mathrm{D}_{2} \mathrm{O}$ gewonnenen Daten legt nahe, daß TMD bei Drücken $>200 \mathrm{MPa}$ niedriger wird als $T_{H}$ und daß somit diese Anomalie des unterkühlten Wassers bei höheren Drücken nicht mehr auftritt.

\subsection{Die isotherme Kompressibilität $\kappa_{T}$}

Die isotherme Kompressibilität einer Flüssigkeit wird durch die Beziehung

$\kappa_{l}-\left(\frac{1}{V}\right)\left(\frac{\partial V}{\partial p}\right)_{T}$

definiert. In allen einfachen Flüssigkeiten nimmt $\kappa_{T}$ mit fallender Temperatur sehr stark ab.

In Fig. 4 ist die Kompressibilität des Wassers bei Atmosphärendruck dargestellt ${ }^{[91.92]}$. Die Daten für den Unterkühlungsbereich wurden von Speedy und Angell ${ }^{[93]}$ in einer dünnen Kapillare gemessen. Bei etwa $320 \mathrm{~K}$, also weit über der Temperatur der maximalen Dichte $(277 \mathrm{~K})$, geht $\kappa_{T}$ durch ein Minimum und steigt dann mit fallender Temperatur steil an. In diesem Diagramm erkennt man sehr deutlich, daß die Anomalien des unterkühlten Wassers eine stetige Fortsetzung der Anomalien im Stabilitätsbereich sind. Zum Vergleich sind die Kompressibilitäten von Methanol $^{[94]}$ und von Glycol ${ }^{[95]} \mathrm{mit}$ in das Diagramm aufgenommen. Kanno und Angell ${ }^{[86.96]}$ gelang es, die Kompressibilitätsmessungen für $\mathrm{H}_{2} \mathrm{O}$ und $\mathrm{D}_{2} \mathrm{O}$ bis $243 \mathrm{~K}$ und $190 \mathrm{MPa}$ auszudehnen. Die Zunahme der Kompressibilität mit fallender Temperatur bleibt bis zu diesen Drücken beobachtbar, ist aber weniger stark ausgeprägt.

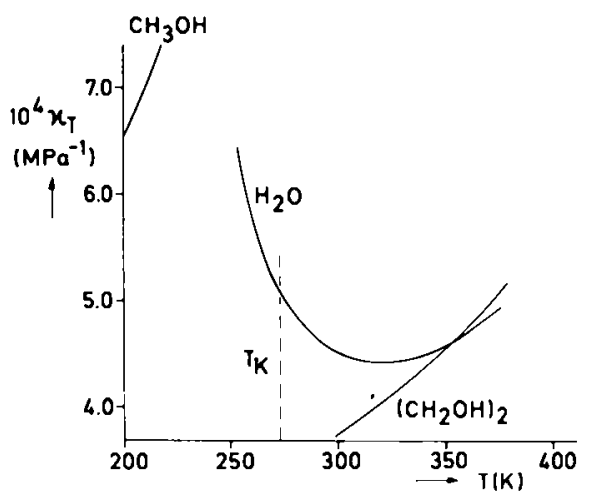

Fig. 4. Temperaturabhängigkeit der isothermen Kompressibilität $\kappa_{r}$ von $\mathrm{H}_{2} \mathrm{O}$ bei $0.1 \mathrm{MPa}$ [91-93]. Zum Vergleich: $\kappa_{T}$ von Methanol [94] und Glycol [95]. $T_{\mathrm{K}}$ : Schmelztemperatur.

\subsection{Die Wärmekapazität bei konstantem Druck $C_{p}$}

Unterkühlt man unter Umgehung der Kristallisation eine Flüssigkeit, die eine deutlich höhere Wärmekapazität (Molwärme) hat als der korrespondierende Kristall, so wird der Flüssigkeit bei weiterer Abkühlung laufend mehr Entropie entzogen als dem Kristall.

Die nicht abgeführte Schmelzentropie wird also aus dem unterkühlten flüssigen System bei weiterer Abkühlung kontinuierlich entfernt. Nach dem 3. Hauptsatz der Thermodynamik ist zu erwarten, daß bei Temperaturerniedrigung die Differenz zwischen den Molwärmen des Kristalls und der metastabilen Flüssigkeit kleiner wird. Bei normalen unterkühlten Systemen wie Alkoholen ${ }^{[97-99]}$ wird dieses auch beobachtet.

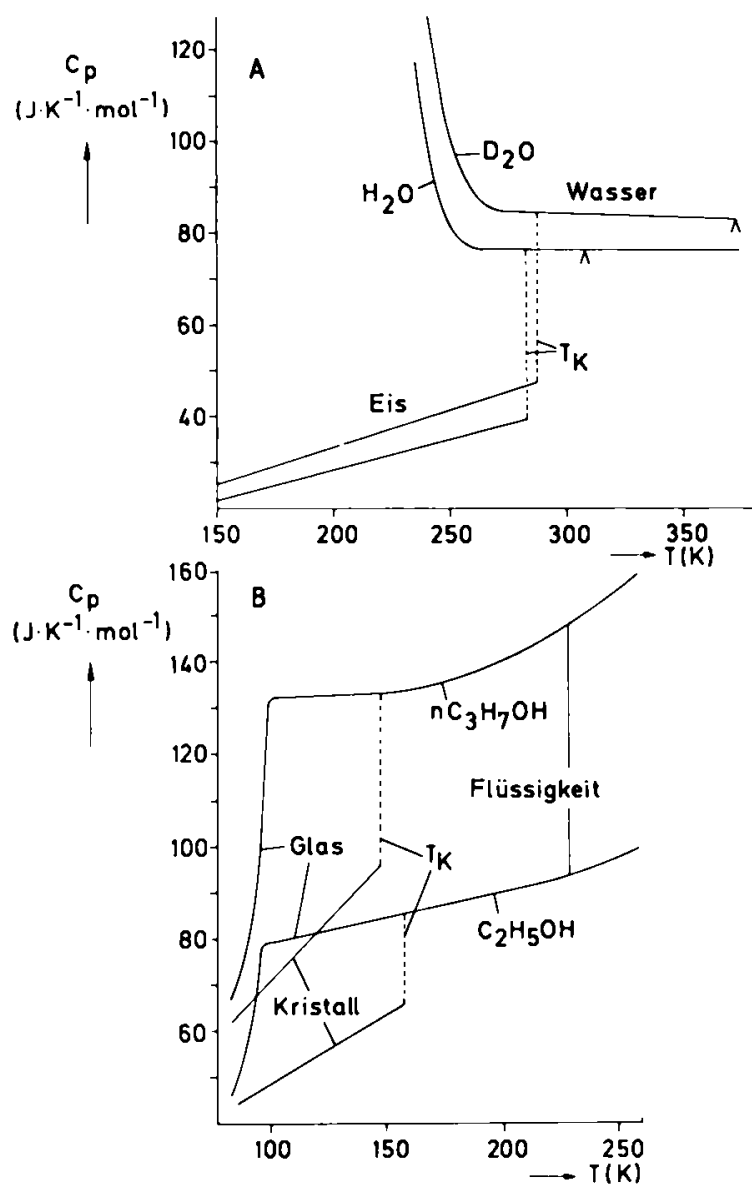

Fig. 5. Temperaturabhăngigkeit der Wärmekapazităt bei konstantem Druck $C_{r}$ A) von $\mathrm{H}_{2} \mathrm{O}$ und $\mathrm{D}_{2} \mathrm{O}$ [100-108], B) von 1-Propanol und Ethanol [97-99] $T_{\mathrm{K}}=$ Schmelztemperatur

Fig. 5 zeigt, daß $C_{p}$ für Ethanol und 1-Propanol mit fallender Temperatur kontinuierlich abnimmt und schließlich in einem engen Intervall im Bereich der Glastemperatur sehr rasch auf den Wert für den Kristall sinkt.

Bei $\mathrm{H}_{2} \mathrm{O}^{\left[100,{ }^{101]}\right.}$ und $\mathrm{D}_{2} \mathrm{O}^{[102,103]}$ steigt $C_{p}^{\mathrm{Liq}}$ ab einer $\mathrm{Mi}$ nimumstemperatur $\left(T_{\min } \mathrm{H}_{2} \mathrm{O}: 308 \mathrm{~K}, \mathrm{D}_{2} \mathrm{O}: 373 \mathrm{~K}\right) \mathrm{mit}$ fallender Temperatur, und dieser Anstieg wird im metastabilen Bereich in der Năhe von $T_{\mathrm{H}}$, der Temperatur der homogenen Nucleation (Fig. 1), sehr steil ${ }^{[104-108)}$. Amorphes festes Wasser, das durch Kondensation von verdünntem $\mathrm{H}_{2} \mathrm{O}$-Dampf im Hochvakuum auf metallische Unterlagen gewonnen wird, hat eine Glastemperatur von $\approx 130-140 \mathrm{~K}^{[109.110]}$. Es ist thermodynamisch unmöglich, daß der Abfall von $C_{p}^{\text {Liq }}$ im unterkühlten Wasser erst in der Nähe dieser Glastemperatur stattfindet. Der wahrscheinliche Verlauf von $C_{p}(I)$ bei unterkühltem Wasser ist daher immer noch Gegenstand von Kontroversen $^{[107.108,111-113]}$. Nach Johari ${ }^{[11]}$ gibt es keine Kontinuität der Zustände zwischen unterkühitem flüssigen Wasser und der amorphen, durch Aufdampfen gewonnenen festen Phase, während Rice et al. ${ }^{[113]}$ die gegenteilige Meinung vertreten. Da es kürzlich gelang, eine Emulsion durch Jet-Kühlung direkt in den „glasartigen“ Zustand umzuwandeln ${ }^{[1 / 4]}$, kann man erwarten, daß kalorische Untersuchungen an dieser Phase diese Streitfrage bald klären werden. 


\subsection{Die statische Dielektrizitätskonstante $\varepsilon_{0}$}

Fig. 6 vergleicht die Temperaturabhängigkeit der statischen Dielektrizitätskonstante $\varepsilon_{0}$ des Wassers im Bereich von 237-373 $\mathrm{K}^{[115,116]}$ mit den entsprechenden Daten von Methanol $^{[117,118]}$ und Ammoniak ${ }^{[19-122]}$. Die Daten für unterkühltes Wasser wurden aus der Dielektrizitätskonstante von Emulsionen mit geringem Wassergehalt errechnet. Verglichen mit dem relativ starken Anstieg von $\varepsilon_{0}$ mit fallender Temperatur bei Methanol zeigt die Kurve für unterkühltes Wasser keine Besonderheiten. Es ist daher unwahrscheinlich, daß unterkühltes Wasser, wie theoretisch für möglich gehalten wurde ${ }^{[123]}$, bei Temperaturen um $220 \mathrm{~K}$ einem ferroelektrischen Übergang zustrebt.

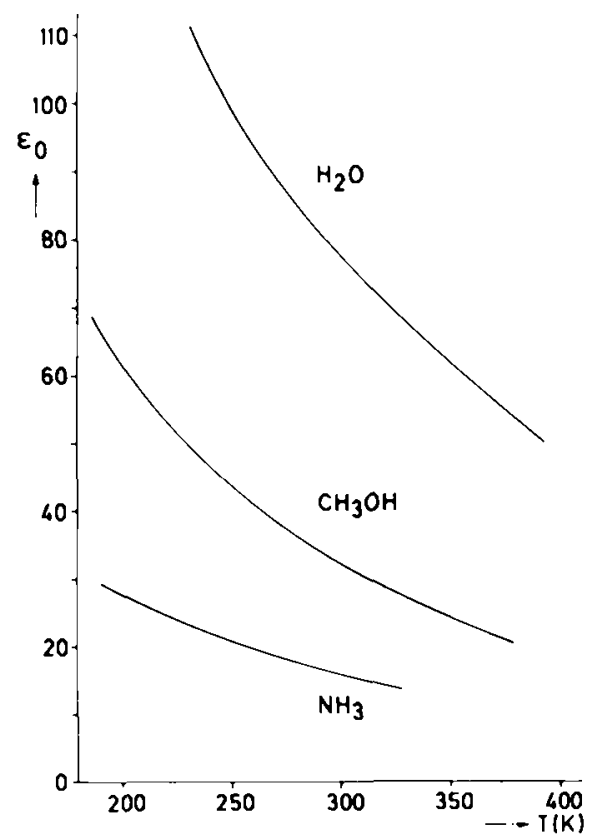

Fig. 6. Temperaturabhăngigkeit der statischen Dielektrizitätskonstante $\varepsilon_{0}$ von $\mathrm{H}_{2} \mathrm{O}$ [115, 116], Methanol [117, 118] und Ammoniak [119-122] bei $0.1 \mathrm{MPa}$.

Die Messung der statischen Dielektrizitätskonstante mittelt über die Dipolorientierungen des Wassermoleküls in einem Bereich, der groß gegenüber den Teilchendurchmessern ist. Das Fehlen von ausgeprägten Anomalien in $\varepsilon_{0}$ gibt einen Hinweis darauf, daß sich die Besonderheiten der Struktur des unterkühlten Wassers nur in relativ kleinen Bereichen, in der Nahordnung, bemerkbar machen.

\subsection{Die Schallgeschwindigkeit $v$}

Von mehreren Arbeitsgruppen wurde die Temperaturabhängigkeit der Ultraschallgeschwindigkeit in flüssigem Wasser bis in den unterkühiten Bereich untersucht ${ }^{1112.124-}$ ${ }^{126]}$. Trinh und $A p f e l^{[127]}$ konnten ihre Messungen bis $240 \mathrm{~K}$ ausdehnen. Die thermodynamische Schallgeschwindigkeit $v(v \rightarrow 0)$ ist gegeben durch:

$$
\begin{aligned}
& 1 / v^{2}=\rho\left[\kappa_{T}-\left(T V \alpha_{p}^{2} / C_{n}\right)\right] \\
& \left(\alpha_{r}=\frac{1}{V}\left(\frac{\partial V}{\partial T}\right)_{n}\right)
\end{aligned}
$$

Aus den zuvor diskutierten Anomalien von $\rho, \kappa_{T}$ und $C_{p}$ folgt, daß sich auch $v$ ungewöhnlich verhalten sollte. Tatsächlich sinkt $v$ bei $T \leq 347 \mathrm{~K}$ mit fallender Temperatur ${ }^{[128]}$, und dieser Abfall scheint sich bis zu den tiefsten Meßtem- peraturen fortzusetzen ${ }^{[129]}$ (Fig. 7). Die Befunde von Bacri et al. ${ }^{1126]}$ legen nahe, daß die Schallgeschwindigkeit mit sinkender Temperatur weiter stark abnimmt und gegen Null geht, während die Untersuchungen von Trinh und $A p$ $\mathrm{fe}^{(127)}$ ein Minimum der Schallgeschwindigkeit bei Temperaturen um $243 \mathrm{~K}$ andeuten. Hier ist darauf hinzuweisen, daß die Messungen am unterkühlten Wasser mit erheblichen experimentellen Schwierigkeiten verbunden sind und bis jetzt nicht so präzise wie Untersuchungen im Stabilitätsbereich sein können.

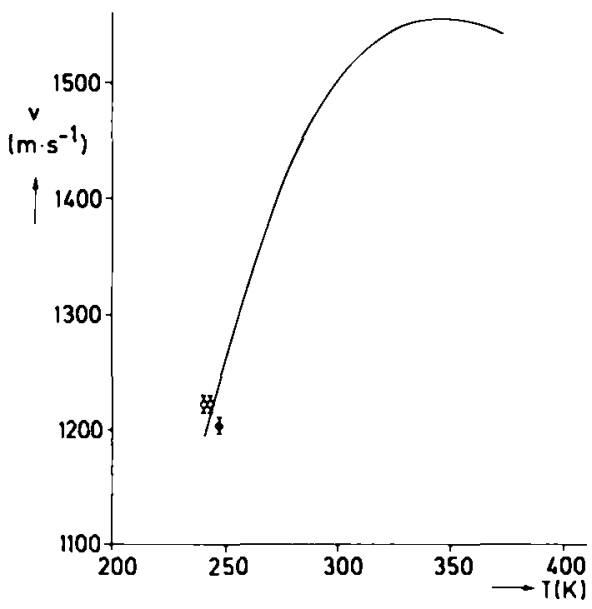

Fig. 7. Temperaturabhăngigkeit der Schallgeschwindigkeit $v$ im unterkühlten Wasser. Nur bei den tiefsten Temperaturen wurden die Meßwerte eingetragen. $\bullet[126]$, o [127].

Dietz et al. ${ }^{[194]}$ haben kürzlich auf der Basis eines Gittermodells die Wärmeleitfähigkeit $\lambda$ von unterkühltem Wasser abgeschätzt. Die Extrapolation der bis $273 \mathrm{~K}$ reichenden Meßergebnisse zu tieferen Temperaturen unter Verwendung der experimentellen $\rho-, C_{p}$ - und $v$-Werte ergibt, $\mathrm{da} \beta \lambda$ bei Temperaturen um $250 \mathrm{~K}$ durch ein Minimum geht und bei weiterer Abkühlung wieder stark ansteigt. Da dieses Minimum deutlich oberhalb von $T_{\mathrm{H}}$ auftritt, der Temperatur der homogenen Nucleation, wäre es interessant, diese Rechnung experimentell zu bestätigen: Es könnte entschieden werden, welches der zur Zeit diskutierten Modelle (siehe Abschnitt 4) das Verhalten des unterkühlten Wassers bei Annäherung an $T_{\mathrm{H}}$ und $T_{\mathrm{S}}$ quantitativ beschreibt ( $T_{\mathrm{S}}$ ist die Singularitätstemperatur, siehe Abschnitt 4.1 und Fig. 12).

\section{Diskussion der statischen Eigenschaften des unterkühlten Wassers}

\subsection{Die Speedy-Angell-Singularität im unterkühlten Wasser}

Die in Abschnitt 3 zusammengefaßten Experimentalbefunde geben Anomalien des Wassers bei konstantem Druck wieder. Diese Anomalien werden weitgehend unterdrückt, wenn man die Eigenschaften bei konstantem Volumen betrachtet. Die Wärmekapazität bei konstantem Volumen $C_{V}$, die aus den schon angegebenen Daten nach

$C_{V}=C_{p}-V T\left(\alpha^{2} / \kappa_{T}\right)$

errechnet werden kann, zeigt zum Beispiel für Atmosphärendruck einen völlig normalen Verlauf. 
Ferner geht aus den Daten hervor, daß hoher hydrostatischer Druck $(p>200 \mathrm{MPa})$ alle Anomalien des Wassers unterdrückt. Also ist die Ursache des anomalen Verhaltens in der Topologie des dreidimensionalen, unregelmäßigen H-Brückennetzwerks zu suchen. Dies zeigt der Zusammenhang, der zwischen den Funktionen $C_{p}, \kappa_{T}, \alpha_{p}$ und den Fluktuationen thermodynamischer Variablen im (TPN)Ensemble ${ }^{[*]}$ besteht:

$\kappa_{T} \sim\left\langle(\delta \bar{V})^{2}\right\rangle, C_{p} \sim\left\langle(\delta S)^{2}\right\rangle$ und $\alpha_{p} \sim\langle\delta \bar{V} \cdot \delta S\rangle$

Die experimentell gefundene Druck- und Temperaturabhängigkeit dieser Größen beruht somit auf Dichte-, Enthalpie- und Entropiefluktuationen, deren Amplituden mit sinkender Temperatur drastisch wachsen. Speedy und $A n-$ gell $^{[93]}$ haben daraus auf die Existenz einer thermodynamischen Singularität im unterkühlten Wasser bei der Temperatur $T_{\mathrm{S}}$ geschlossen. $T_{\mathrm{S}}$ liegt nur wenig unter der Temperatur der homogenen Nucleation $T_{\mathrm{H}}$ und wird wie diese mit steigendem hydrostatischen Druck zu tieferen Temperaturen verschoben (siehe Fig. 12). Die Autoren interpretieren $T_{\mathrm{S}}$ als eine Grenze der mechanischen Instabilität der unterkühlten Flüssigkeit. Diese Interpretation impliziert ein Divergieren der thermodynamischen Antwortfunktionen bei Annäherung an $T_{S}(p)$. Molekulare Ursache dieser anomalen Fluktuationen soll die Bildung geordneter Bereiche des tetraedrischen Netzwerks mit linearen H-Brücken sein.

Hydrostatischer Druck erschwert die Bildung linearer H-Brückenbindungen, d. h. die Bildung der Konfiguration mit niedrigster Energie, aber größtem Platzbedarf. Druckanwendung führt somit zur Abnahme der Amplituden der anomalen Fluktuationen und zur rapiden Annäherung der Temperatur maximaler Dichte (TMD) an $T_{H}(p)$ (siehe Fig. 1). Gegenwärtig gibt es zwei (siehe auch Abschnitt 10) theoretische Modellbetrachtungen zu den anomalen Eigenschaften unterkühlten flüssigen Wassers. Das Perkolationsmodell von Stanley ${ }^{[130]}$ et al. (siehe Abschnitt 4.2) assoziiert die vermutete thermodynamische Singularität mit einer Perkolationsschwelle für vierfach koordinierte Wassermoleküle im unregelmäßigen H-Brückennetzwerk. Dagegen zeigen Stillingers ${ }^{[131]}$ Überlegungen zur statistischen Mechanik endlicher Systeme, daß genügend starke Unterkühlung zu einer "thermodynamischen Katastrophe“ führen kann.

\subsection{Das Perkolationsmodel}

Stanley et al. ${ }^{[130.132 .133]}$ entwickelten auf der Basis der "correlated site percolation“-Theorie ${ }^{[130]}$ ein Konzept, das die beobachteten Anomalien qualitativ erklärt und darüber hinaus in quantitativer Übereinstimmung mit ComputerSimulationen an Modellwasser ist. Die Wassermoleküle werden in fünf Spezies, entsprechend der Zahl ihrer intakten $\mathrm{H}$-Brücken, eingeteilt und ein Bruchteil $\mathrm{p}_{\mathrm{B}}$ intakter $\mathrm{H}$ Brücken willkürlich zwischen ihnen verteilt. Der Bruchteil $f_{j}$ an Molekülen der Spezies j beträgt dann nach dem Binomialgesetz

$f_{j}=\left(\begin{array}{l}4 \\ j\end{array}\right) p_{\mathbf{B}}^{4}\left(1-p_{B}\right)^{4-1}$

[*] Unter dem (TPN)-Ensemble versteht man: thermodynamische Vielteilchensysteme, bei denen die Temperatur $T$, der Druck $p$ und die Teilchenzahl $N$ konstant gehalten werden.
Wegen $f_{4}=p_{B}^{4}$ hat das Modell einen eingebauten Verstärkungsmechanismus, der mit wachsendem $p_{B}$ ( $\triangleq$ sinkender Temperatur) den Bruchteil der vierfach koordinierten Moleküle überproportional anwachsen läßt.

Während also intakte $\mathrm{H}$-Brücken regellos zwischen den Molekülen verteilt angenommen werden, ergibt sich $z$. B. für Moleküle der Spezies $\mathrm{j}=4$ eine Neigung zur Clusterbildung. Die lokalen physikalischen Eigenschaften der Cluster von Molekülen mit der Koordinationszahl 4 lassen sich natürlich nicht aus dem Modell ableiten, sondern müssen als plausible Annahmen eingeführt werden. Eine Analyse der Ergebnisse von Computer-Simulationen zeigt, daß Dichte und Entropie der Cluster von vierfach koordinierten Wassermolekülen geringer sind als die mittleren Werte der Flüssigkeit. Damit werden die Dichte- und Entropiefluktuationen größer als in einem homogenen Netzwerk.

Ein direkter experimenteller Nachweis der Dichtefluktuationen gelingt mit einem Streuexperiment, da der Strukturfaktor $S(q)$ einer Flüssigkeit der Fourier-Transformierten der Dichtekorrelationsfunktion proportional ist ${ }^{134]}$. Das Anwachsen der Korrelationslänge $\boldsymbol{\xi}$ der Dichtefluktuationen führt zu einem Anwachsen von $S(q)$ für $q \rightarrow 0$, also einer Zunahme der Streuintensität bei kleinen Streuwinkeln. Bosio. Teixeira und Stanley ${ }^{[135]}$ haben Kleinwinkel-Röntgenstreuexperimente in unterkühltem $\mathrm{H}_{2} \mathrm{O}$ und $\mathrm{D}_{2} \mathrm{O}$ durchgeführt, die tatsächlich ein Anwachsen der Streuintensität bei kleinen Streuwinkeln anzeigen. Die Korrelationslänge $\xi$ der Dichtefluktuationen beträgt bei $T=253 \mathrm{~K}$ ungefähr $8 \AA$.

\subsection{Das differentialgeometrische Modell $^{[131]}$}

Dieses Modell geht von der Zustandssumme $Z_{N}$ im (TPN)-Ensemble aus:

$Z_{\mathrm{n}}(\beta . p)=Z_{\mathrm{N}}^{(0)}(\exp (-\beta \theta)\rangle_{\mathrm{TPN}} \quad ; \quad \beta=k T^{-1}$

wobei $\theta$ die Summe aus der potentiellen Energie $\phi$ der Wassermoleküle und dem Produkt $p \cdot V$ ist. Es wird angenommen, daß sich die molekularen Konfigurationen durch zwei unabhängige Parameter, die potentielle Energie $\phi$ und den strukturellen Ordnungsparameter $\psi$ beschreiben lassen. $\psi$ bezeichnet die Anzahl gebundener bicyclischer Octamere, die von einer molekulardynamischen Rechnung mit dem Polarisationsmodell als globales Minimum im Konfigurationsraum identifiziert wurden ${ }^{[63]}$. Ein solches System hat bei tiefen Temperaturen zwei Zustände, die durch großes $\psi$ und kleines $\theta$ charakterisierte feste Phase und die flüssige Phase, die durch großes $\theta$ und kleines $\psi$ gekennzeichnet ist.

In diesem System kann es bei Abkühlung zu einer Katastrophe kommen, das heißt, daß für die flüssige Phase der Ordnungsparameter $\psi$ divergieren kann. Gerade diese Situation könnte im unterkühlten Wasser bei der Singularitätstemperatur $T_{\mathrm{S}}$ eintreten. Da $\psi$ die Anzahl gebundener bicyclischer Octamere mißt und es sich dabei um offene Strukturen mit linearen $\mathrm{H}$-Brücken im unregelmäßigen $\mathrm{H}$ Brückennetzwerk handelt, sind mit den Fluktuationen des Ordnungsparameters auch Dichtefluktuationen im $\mathrm{H}$ Brückennetzwerk assoziiert. Divergente Dichtefluktua- 
tionen bedingen aber eine Divergenz der Funktionen $\kappa_{r}$, $\alpha_{p}$ und $C_{p}$.

Aus dem differentialgeometrischen Modell und dem Perkolationsmodell läßt sich ableiten, daß durch die Aggregation geordneter Bereiche oder analog dazu durch die Perkolation von Clustern vierfach koordinierter Moleküle eine irreversible Situation geschaffen wird, die zur Zerstörung der flüssigen Phase, zur homogenen Nucleation, führt. Es wird also experimentell sehr schwer sein, die Temperaturabhängigkeit physikalischer Eigenschaften beliebig nahe an $T_{\mathrm{S}}$ heran zu messen.

Ein wesentlicher Unterschied der besprochenen Modelle soll hier noch einmal herausgestellt werden: Während die Überlegungen von Speedy und Angell ${ }^{[93]}$ sowie Stillinger ${ }^{[131]}$ (Abschnitt 4.1) zu einer Divergenz der statischen Eigenschaften $\left(\kappa_{r}, C_{p}, \alpha_{p}\right)$ bei Annäherung an $T_{\mathrm{S}}$ führen, sollte im Perkolationsmodell von Stanley und Tei$x_{\text {xeira }}{ }^{[132]}$ (Abschnitt 4.2) keine solche Divergenz auftreten.

Wie Bosio, Teixeira und Stanley ${ }^{[135]}$ betonten, könnte im Prinzip mit Streuexperimenten bei sehr kleinen Streuwinkeln oberhalb von $T_{\mathrm{H}}$ untersucht werden, ob die Korrelationslänge $(\xi)$ der Dichtefluktuationen bei $T_{\mathrm{S}}$ divergiert oder oberhalb $T_{\mathrm{H}}$ durch ein Maximum geht.

\section{Dynamische Eigenschaften des flüssigen Wassers}

Dynamische Eigenschaften von Flüssigkeiten werden in der statistischen Mechanik durch Zeitkorrelationsfunktionen dynamischer Variablen ${ }^{[136]}$ beschrieben. Die theoretische Berechnung der Zeitkorrelationsfunktionen molekularer Flüssigkeiten ist außerordentlich kompliziert. Durch Computer-Simulationen sind jedoch für Modellwasser viele dieser Funktionen explizit berechnet worden.

\subsection{Molekulardynamische Rechnungen}

Dynamische Größen können nur mit der molekulardynamischen Methode berechnet werden. Im Gegensatz zu den strukturellen Aspekten des flüssigen Wassers sind seine dynamischen Eigenschaften nur in wenigen Fällen durch Computer-Simulationen untersucht worden.

Platzwechselvorgänge in Flüssigkeiten werden in der statistischen Mechanik durch Geschwindigkeits-Autokorrelationsfunktionen $\varphi(t)=\langle\vec{v}(0) \vec{v}(t)\rangle$ beschrieben. Die Geschwindigkeitskorrelationsfunktion für den Massenschwerpunkt der Moleküle $\varphi_{\mathrm{CM}}(t)$ wurde über einen weiten Temperaturbereich für „BNS-Wasser"[37, 38] und „ST2Wasser $“[40.41]$ ) berechnet. Bei tiefen Temperaturen zeigt sie bei $t<0.5$ ps ausgeprägte Oszillationen um $\varphi_{\mathrm{CM}}(t)=0$. Die spektrale Dichtefunktion hat bei tiefen Temperaturen Maxima bei $\approx 40-60$ und $190-225 \mathrm{~cm}^{-1}$, die sich mit steigender Temperatur zu niedrigen Frequenzen verschieben. Diese Banden sind als $\mathrm{O}-\mathrm{O}-\mathrm{O}-\mathrm{Biege-}$ bzw. $\mathrm{O}-\mathrm{O}$ Streckschwingungen identifiziert worden. Trotz der - verglichen mit einfachen Flüssigkeiten - ausgeprägten Oszillationen sind diese so stark gedămpft, daß $\varphi(t)$ nach etwa 1 ps auf Null abgeklungen ist. Wie Rahman und Stillinger betonten, implizieren die Oszillationen von $\varphi_{\mathrm{CM}}(t)$ keinen sprunghaften Platzwechsel in diskrete Gleichgewichtslagen. Vielmehr erfolgt der Platzwechsel von Wassermolekülen als kontinuierlicher Prozeß während der ständigen
Umordnung des labilen, ungeordneten H-Brückennetzwerks. Ein $M a ß$ für die diffusive Schwerpunktsbewegung in einer homogenen Flüssigkeit ist der Selbstdiffusionskoeffizient $D$. Er kann mit einer Green-Kubo-Beziehung durch die Korrelationsfunktion $\varphi(t)$ ausgedrückt werden:

$D=(1 / 3) \int_{0}^{\infty}\langle\vec{v}(0) \cdot \vec{v}(\tau)) d \tau$

Die Temperaturabhängigkeit von $D$ ist für „BNS-Wasser“ und „ST2-Wasser" deutlich schwächer als experimentell gefunden wird. Werden Wechselwirkungen mit großer Reichweite durch Einbeziehung eines Reaktionsfeldes berücksichtigt, so ist der berechnete Diffusionskoeffizient nahezu doppelt so groß wie der experimentelle Wert ${ }^{[137]}$. Im Gegensatz zu Modellpotentialen für starre Moleküle ergeben molekulardynamische Rechnungen mit CF-Potentialen $^{\text {[5s] }}$ Diffusionskoeffizienten, die bei Raumtemperatur um etwa einen Faktor 2 bis 3 zu niedrig sind. Rapaport und Scheraga ${ }^{[501}$ haben molekulardynamische Rechnungen mit dem MCY-Potential durchgeführt. Die dabei ermittelten Diffusionskoeffizienten sind für leicht unterkühltes Wasser um $\approx 67 \%$ zu hoch und bei $377 \mathrm{~K} \mathrm{um} \approx 12 \%$ zu klein. Wie schon für „BNS-Wasser" und „ST2-Wasser" ist die Temperaturabhängigkeit zu gering. Dies zeigt, daß diese Modellpotentiale nicht imstande sind, den beobachteten starken Abfall des Diffusionskoeffizienten im unterkühlten Wasser richtig zu beschreiben. Die einzige Bestimmung des Selbstdiffusionskoeffizienten $D$ aus molekulardynamischen Rechnungen für „ST2-Wasser" unter hohem Druck $^{[43]}$ ergab bei einer Dichteerhöhung um 35\% eine Verringerung von $D$ um $\approx 10 \%$ bei $370 \mathrm{~K}$. Beobachtet wird diese Reduktion bereits bei $\approx 6 \%$ Dichteerhöhung. Daraus muß geschlossen werden, daß „ST2-Wasser“ wesentlich schwächere Druckeffekte zeigt als reales Wasser. Abzuwarten bleibt, ob sich mit molekulardynamischen Simulationen die bei tiefen Temperaturen und mittleren Drücken ( $<250 \mathrm{MPa}$ ) beobachtete Zunahme von $D$ mit dem Druck realisieren läßt.

Neben Translationsbewegungen sind bei Molekülen auch Rotationsbewegungen möglich. Diese werden in der statistischen Mechanik durch die Funktionen

$G_{1}(t)=\left\langle P_{1}\left[\vec{u}_{\mathrm{j}}(t) \cdot \vec{u}_{j}(0)\right]\right\rangle$

beschrieben, die die Orientierungskorrelation eines Einheitsvektors $\overrightarrow{\boldsymbol{u}}$ im Molekül $\mathrm{j}$ nach verschiedenen Zeiten angeben. Die Funktionen für $l=1,2$ sind von besonderem Interesse, da sie eng mit meßbaren Größen verknüpft sind ${ }^{[138]}$. Rahman und Stillinger ${ }^{[37,38]}$ haben die Orientierungsrelaxation von Wassermolekülen in molekulardynamischen Rechnungen mit dem BNS-Potential untersucht. Sowohl $G_{1}(t)$ also auch $G_{2}(t)$ zeigen für $t<0.1$ ps ein Librationsverhalten. Für größere Zeiten klingen beide Funktionen nahezu exponentiell ab. Die effektiven Zeitkonstanten $\tau_{1}$ und $\tau_{2}$ des exponentiellen Abfalls werden mit sinkender Temperatur größer. $\tau_{1}$ und $\tau_{2}$ sind von van Gunsteren et al. ${ }^{137 !}$ für „ST2-Wasser" mit Reaktionsfeid ebenfalls bestimmt worden, während Rapaport et al. ${ }^{(50)} \tau_{1}$ mit dem MCY-Potential berechneten. Beide Gruppen erhalten wesentlich kleinere Zeitkonstanten als für „BNS-Wasser“ gefunden wurde ${ }^{[37]}$, das die beste Übereinstimmung dieser 
Größen mit dem Experiment liefert. Die berechnete Temperaturabhängigkeit stimmt mit Ausnahme der Werte für unterkühltes Wasser gut mit der gemessenen überein.

Das Librationsverhalten der Moleküle wurde von Rahman und Stillinger ${ }^{[37.38]}$ mit Winkelgeschwindigkeits-Autokorrelationsfunktionen

$f_{r x}(t)=\left\langle\omega_{r}(0) \cdot \omega_{r a}(t)\right\rangle \quad ; \quad \alpha=\mathrm{x}, \mathrm{y}, \mathrm{z}$

näher untersucht. Eine schwache Zeitabhängigkeit von $f_{\alpha}(t)$ bedeutet eine relativ unbehinderte Rotation, wăhrend rasche Oszillationen der Funktion darauf hinweisen, daß das Molekül durch seine Nachbarn in einer bestimmten Orientierung fixiert wird. Alle drei Funktionen zeigen starke Oszillationen, die jedoch sehr schnell $\left(<10^{-13} \mathrm{~s}\right.$ bei $307 \mathrm{~K})$ abklingen. Die Nachbarmoleküle sind demnach nicht imstande, ein „Käfig-Molekül“" länger als $10^{-13} \mathrm{~s}$ in seiner Orientierung festzuhalten. Mit steigender Temperatur werden die Oszillationen schwächer, die „Periodendauer" wird größer.

\subsection{Statistische Mechanik und Hydrodynamik}

1978 haben $C h u$ und Sposito ${ }^{[139]}$ den Versuch unternommen, die Schwerpunktsbewegung der Wassermoleküle mit einer zeitabhängigen Störungstheorie im Rahmen der klassischen statistischen Mechanik zu beschreiben. Die Autoren gehen vom ,relaxing cage"-Modell[140] der dynamischen Struktur einer atomaren Flüssigkeit aus und verallgemeinern es für den Fall flüssigen Wassers. Als ungestörtes Referenzsystem wird dabei Eis Ih gewählt, dessen Gitterdynamik die Eigenmoden des Systems liefert. Zur Berechnung der Geschwindigkeitskorrelationsfunktion

$\varphi(t)=\langle\vec{v}(0) \vec{v}(t)\rangle$

wird angenommen, daß zu einem beliebigen Zeitpunkt $t=0$ in der Flüssigkeit eine dem Eis entsprechende Nahordnung vorliegt. Diese Nahordnung soll die gleichen Eigenschwingungen haben wie Eis Ih. Ihre Zerstörung durch die thermische Bewegung der Nachbarmoleküle bedingt ein zeitliches Abklingen der Eigenmoden. Stöße mit Nachbarteilchen führen zu einem stetigen Verlust der Korrelation der Teilchengeschwindigkeit. Während nach sehr kurzen Zeiten die Käfigstruktur noch weitgehend erhalten ist und das betrachtete Molekül oszilliert, wird aufgrund der thermischen Bewegung der Nachbarn die Nahordnung aufgelöst, und die oszillierende Bewegung des betrachteten Moleküls geht in eine diffusive Bewegung über. Die spektrale Dichtefunktion dieses Modells kann durch geeignete Wahl der Lebensdauern der Eigenmoden das aus molekulardynamischen Rechnungen erhaltene Leistungsspektrum in groben Zügen reproduzieren.

In einer anschließenden Arbeit zeigt Sposito ${ }^{[141]}$ anhand von Literaturdaten für den Selbstdiffusionskoeffizienten $D$, die dielektrische Relaxationszeit $\tau_{\mathrm{d}}$ und die NMROrientierungskorrelationszeit $\tau_{2}$, daß die Einteilchenbewegung in flüssigem Wasser bei niedrigen Drücken und Temperaturen mit einem hydrodynamischen Modell adäquat beschrieben werden kann. In der hydrodynamischen Näherung gilt mit $f=\beta \cdot a / 3 \eta$ :
$D=\frac{k_{\mathrm{B}} T}{4 \pi \eta a}(1+f) /(1+3 / 2 f)$

$\tau_{\mathrm{d}} \simeq \tau_{1}=\frac{4 \pi \eta a^{3}}{k_{\mathrm{B}} T} f /(1+f)$

$t_{2}=\frac{4 \pi \eta a^{3}}{3 k_{\mathrm{B}} T} f /(1+f)$

wobei $\eta$ die Scherviskosität, $a$ der van-der-Waals-Radius der als kugelförmig betrachteten Wassermoleküle ist und der Parameter $\beta$ die Randbedingungen an der Kugeloberfläche charakterisiert ${ }^{[142]}$. Die Gleichungen implizieren $\tau_{1} / \tau_{2}=3$ und somit die Gültigkeit des Rotationsdiffusionsmodells ${ }^{[143]}$. Mit gemessenen Viskositätsdaten und $a=1.44$ Ả können die Meßwerte von $D, \tau_{1}$ und $\tau_{2}$ sehr gut reproduziert werden, sofern für die translatorische Diffusion $\beta \simeq 0$ und für die rotatorische Diffusion $\beta \rightarrow \infty$ angenommen wird.

\section{Transport- und Relaxationseigenschaften des unterkühlten Wassers}

\subsection{Die Scherviskosität $\eta$}

Die Viskosität $\eta$ beschreibt den Impulstransport in einer Flüssigkeit. Zu diesem tragen kinetische Prozesse und zwischenmolekulare Kräfte bei. Wie theoretische Überlegungen zeigen, faßt die Viskosität die Auswirkungen kurzlebiger Prozesse, die etwa auf der Zeitskala einer mittleren Stoßzeit $\tau_{c}{ }^{[144]}$ abklingen, auf die Dynamik des Systems bei langen Zeiten zusammen.

Experimentelle Untersuchungen der Temperatur- und Druckabhängigkeit der Scherviskosität $\eta$ sind im thermodynamisch stabilen Bereich der Flüssigkeit mit großer Genauigkeit durchgeführt worden ${ }^{[145-147]}$. Die starke Temperaturabhängigkeit der Viskosität im kalten Wasser wird in der metastabilen unterkühlten Phase noch verstärkt. Bei Normaldruck konnte $\eta$ bis $238 \mathrm{~K}$ für $\mathrm{H}_{2} \mathrm{O}$ und $242 \mathrm{~K}$ für $\mathrm{D}_{2} \mathrm{O}$ bestimmt werden ${ }^{[148.149]}$ (Fig. 8).

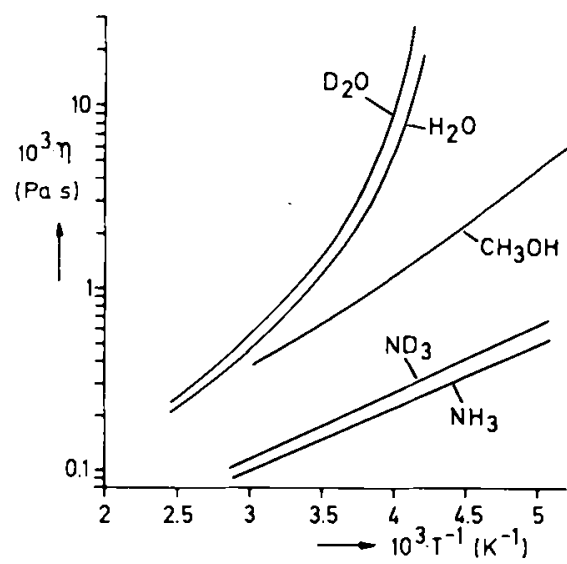

Fig. 8. Temperaturabhängigkeit der Scherviskosităt $\eta$ von $\mathrm{H}_{2} \mathrm{O}, \mathrm{D}_{2} \mathrm{O}[145$ 149], Methanol [188], $\mathrm{NH}_{3}$ und $\mathrm{ND}_{3}$ [184-187].

Die Druckabhängigkeit der Viskosität zeigt in kaltem Wasser $\left(\mathrm{H}_{2} \mathrm{O}: T \leq 312 \mathrm{~K}, \mathrm{D}_{2} \mathrm{O}: T \leq 317 \mathrm{~K}^{[150 l}\right)$ anomales Verhalten, da - im Gegensatz zu einfachen Flüssigkeiten $\eta$ mit steigendem Druck abnimmt und durch ein Minimum geht, das mit fallender Temperatur zu höheren Drücken 
verschoben und zunehmend schärfer wird ${ }^{[151.152]}$. Messungen der Viskosität in der metastabilen Phase bei Drücken $p>0.1 \mathrm{MPa}$ sind nicht bekannt. Eine Interpolation der Meßwerte bei hohen Drücken und der Normaldruckwerte für die unterkühlte Phase bei $258 \mathrm{~K}$ deutet jedoch in diesem Bereich auf eine starke Abnahme der Viskosität mit steigendem Druck hin. Der Quotient $\left[\left(\eta\left(\mathrm{D}_{2} \mathrm{O}\right)\right) /\left(\eta\left(\mathrm{H}_{2} \mathrm{O}\right)\right)\right]_{T, p}$ nimmt mit fallender Temperatur rasch zu.

\subsection{Der Selbstdiffusionskoeffizient $D$}

Der Selbstdiffusionskoeffizient $D$ kennzeichnet unmittelbar die Schwerpunktsbewegung der Teilchen. Im thermodynamisch stabilen Bereich der flüssigen Phase sind zahlreiche Messungen der Temperatur- und Druckabhängigkeit von $D$ in $\mathrm{H}_{2} \mathrm{O}$ und $\mathrm{D}_{2} \mathrm{O}$ durchgeführt worden ${ }^{1145.146]}$. Messungen der Temperaturabhängigkeit des Diffusionskoeffizienten gibt es im metastabilen unterkühlten Wasser nur bei Normaldruck bis $242 \mathrm{~K}^{[153]}$ (siehe Fig. 9).

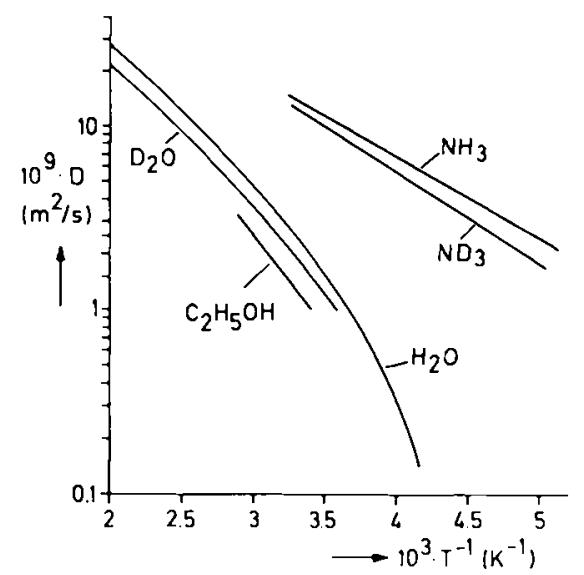

Fig. 9. Temperaturabhängigkeit des Selbstdiffusionskoeffizienten $D$ von $H_{2} \mathrm{O}, \mathrm{D}_{2} \mathrm{O}[145,146,153-156]$, Ethanol [192], $\mathrm{NH}_{3}$ und $\mathrm{ND}_{3}[184-191]$.

Wie die Viskosität $\eta$ hat auch $D$ eine starke Temperaturabhängigkeit ${ }^{[154]}$, die im unterkühlten Wasser noch ausgeprägter wird ${ }^{[155]}$. Mit wachsendem hydrostatischen Druck wird die Temperaturabhängigkeit schwächer ${ }^{[155]}$. Im kalten Wasser durchläuft der Diffusionskoeffizient mit steigendem Druck ein Maximum ${ }^{[154-156]}$, das mit sinkender Temperatur zu höheren Drücken verschoben wird ${ }^{[152]}$.

\subsection{Die Spin-Gitter-Relaxationsgeschwindigkeit $R_{1}$}

Die Messung der kernmagnetischen Spin-Gitter-Relaxationsgeschwindigkeit $R_{1}$ ist eine Möglichkeit, Informationen über molekulare Bewegungen im flüssigen Wasser zu erhalten. Die Anwendung einer halbklassischen Theorie ergibt ${ }^{[157]}$ :

$R_{1}=\frac{1}{T_{1}} \sim \sum_{m=1.2} c(\mathrm{~m}) J^{(m)}\left(\mathrm{m} \omega_{0}\right)$

Hierin sind $T_{1}$ die experimentell bestimmte Spin-GitterRelaxationszeit und $J\left(\omega_{0}\right)$ die spektrale Dichte von Autokorrelationsfunktionen $G(t)$ bei der Meßfrequenz $\omega_{0}$. Die Faktoren $c(\mathrm{~m})$ kennzeichnen Art und Stärke der Wechsel- wirkung zwischen Spinsystem und Gitter. Die Zeitabhängigkeit der Fluktuationsfunktion $G(t)$ kann häufig durch eine mikroskopische Korrelationszeit $\tau$ gekennzeichnet werden, die die zugrundeliegenden molekularen Bewegungen charakterisiert.

Relaxationszeiten $T_{1}$ sind für die Isotope ${ }^{1} \mathrm{H},{ }^{2} \mathrm{H}$ und ${ }^{17} \mathrm{O}$ in Wasser über einen großen Druck- und Temperaturbereich gemessen worden ${ }^{158-1661}$. Insbesondere ist die Protonen- und Deuteronen-Spin-Gitter-Relaxationszeit die einzige physikalische Größe, die im gesamten metastabilen Bereich der flüssigen Phase bis zu Drücken von $300 \mathrm{MPa}$ und bei Temperaturen $T \geq T_{\mathrm{H}}$ (Fig. 10) gemessen werden konnte $^{[164.165]}$.

Anders als bei einfachen schwach-oder nicht-assoziierten Flüssigkeiten ${ }^{[167]}$ zeigt $T_{1}$ bei Wasser im Arrhenius-Diagramm eine nicht-lineare Temperaturabhängigkeit, die in der unterkühlten flüssigen Phase besonders stark ist. Hydrostatischer Druck führt zu einer schwächeren isobaren Temperaturabhängigkeit von $T_{1}{ }^{[165]}$. Die isotherme Druckabhängigkeit von $T_{1}$ in kaltem Wasser hat - ebenso wie bei $\eta$ und $D$ - einen Extremalwert ${ }^{[168]}$. In der unterkühlten Phase wird diese Anomalie im Bereich niedriger Drücke $(p<200 \mathrm{MPa})$ mit sinkender Temperatur wesentlich ausgeprägter als im thermodynamischen Stabilitätsbereich der Flüssigkeit ${ }^{[164-167]}$. Bei sehr tiefen Temperaturen $(T<220$ K) und - wegen der Druckabhängigkeit der Temperatur der homogenen Nucleation $T_{\mathrm{H}}$ notwendigerweise - hohen Drücken nimmt $T_{1}$ dagegen wie in einfachen Flüssigkei$\operatorname{ten}^{[167,169-171]}$ monoton mit steigendem Druck ab.

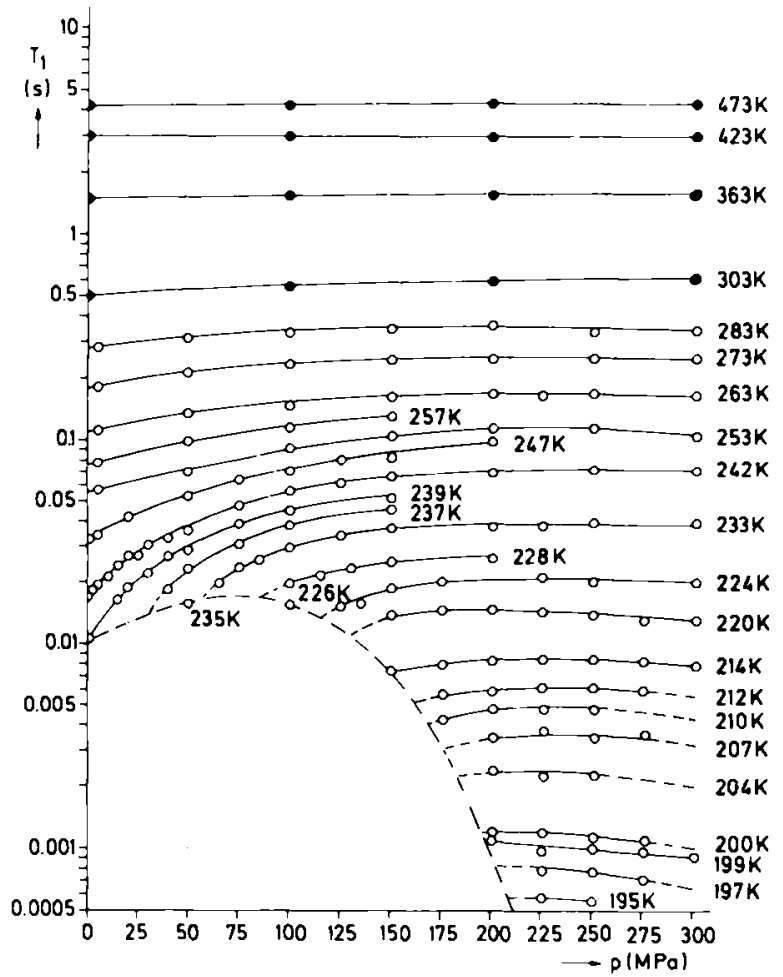

Fig. 10. Druckabhängigkeit von Isothermen der Spin-Gitter-Relaxationszeit $T_{1}$ der Deuteronen in $\mathrm{D}_{2} \mathrm{O} . \bullet[151,152], \circ[165]$.

Nahe $T_{\mathrm{H}}$ konnte im Falle der Protonen- und Deuteronenrelaxation ein Minimum in der $T,(T)$-Kurve nachgewiesen werden (Fig. 11). Dieses tritt auf, wenn die mikroskopische Zeitkonstante $\tau$ mit der Meßfrequenz vergleichbar wird $\left(\omega_{0} \cdot \tau \simeq 1\right)$, da bei hohen Temperaturen $R_{1} \sim \tau$ und bei tiefen Temperaturen $R_{1} \sim\left(\omega_{0}^{2} \tau\right)^{-1}$ gilt und $\tau$ mit fallen- 


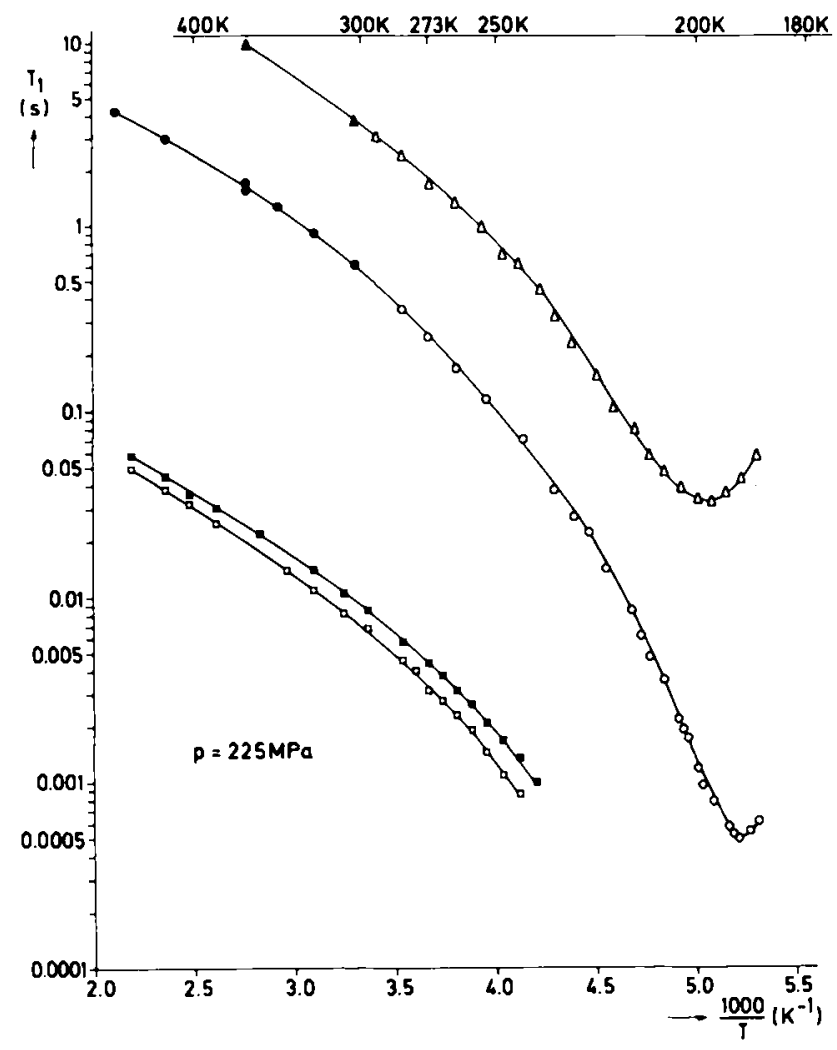

Fig. 11. Temperaturabhängigkeit von 225-MPa-Isobaren der Spin-Gitter-Relaxationszeit $T_{1}, \Delta[193], \Delta[164]\left({ }^{1} \mathrm{H}-T_{1}\right.$ in $\left.\mathrm{H}_{2} \mathrm{O}\right) ; \bullet$ [193], o [165] ( ${ }^{2} \mathrm{H}-T_{1}$ in $\left.\mathrm{D}_{2} \mathrm{O}\right) ; \quad[166]\left({ }^{17} \mathrm{O}-T_{1}\right.$ in $\left.\mathrm{H}_{2} \mathrm{O}\right)$ : $\mathrm{a}[166]\left({ }^{17} \mathrm{O}-T_{1}\right.$ in $\left.\mathrm{D}_{2} \mathrm{O}\right)$.

der Temperatur monoton steigt. Frequenzabhängige Messungen der Relaxationszeit $T_{1}$ in diesem Temperaturbereich bieten die Möglichkeit, die Form der spektralen Dichtefunktion $J\left(m \omega_{0}\right)$ zu bestimmen. Eine Interpretation der Meßergebenisse wird in Abschnitt 7 mit den aus den gemessenen Daten errechneten mikroskopischen Korrelationszeiten $\tau$ versucht.

\section{Diskussion der dynamischen Eigenschaften des unterkühlten Wassers}

Wie die Diskussion der statischen und dynamischen Eigenschaften des flüssigen Wassers gezeigt hat, verhält sich Wasser unter hohem hydrostatischen Druck wie eine normale viskose Flüssigkeit ${ }^{[172]}$. Die isobare Temperaturabhängigkeit dynamischer Eigenschaften vieler viskoser Flüssigkeiten läßt sich quantitativ mit der Vogel-Tammann-Fulcher-Gleichung (10) ${ }^{[173-175]}$ beschreiben:

$X=X_{0} \exp \left(\frac{B}{T-T_{0}}\right)($ VTF-Gleichung $)$

Nach dieser Gleichung divergiert die dynamische Größe $X$ bei Annäherung an die Temperatur $T_{0}$. Dies ist bei viskosen Flüssigkeiten dann der Fall, wenn die Flüssigkeit in einen als Glaszustand bezeichneten amorphen festen $\mathrm{Zu}$ - stand übergeht, bei dem alle molekularen Umlagerungsprozesse eingefroren sind. $T_{0}$ wird deshalb als ideale Glastemperatur bezeichnet. Die isobare Temperaturabhängigkeit der Orientierungskorrelationszeit $\tau_{2}$ in flüssigem Wasser unter hohem hydrostatischen Druck ( $p \geq 200 \mathrm{MPa}$ ) läßt sich sehr gut mit der VTF-Gleichung beschreiben ${ }^{[165,166]}$. Die bei der Anpassung der experimentellen Werte von $\tau_{2}$ an die VTF-Gleichung erhaltenen Parameter $\tau_{0}, B, T_{0}$ sind in Tabelle 1 zusammengefaßt. Die gefundenen Glastemperaturen $T_{0}(p)$ zeigen eine schwach positive, lineare Druckabhängigkeit (Fig. 12). Die auf Normaldruck extrapolier-

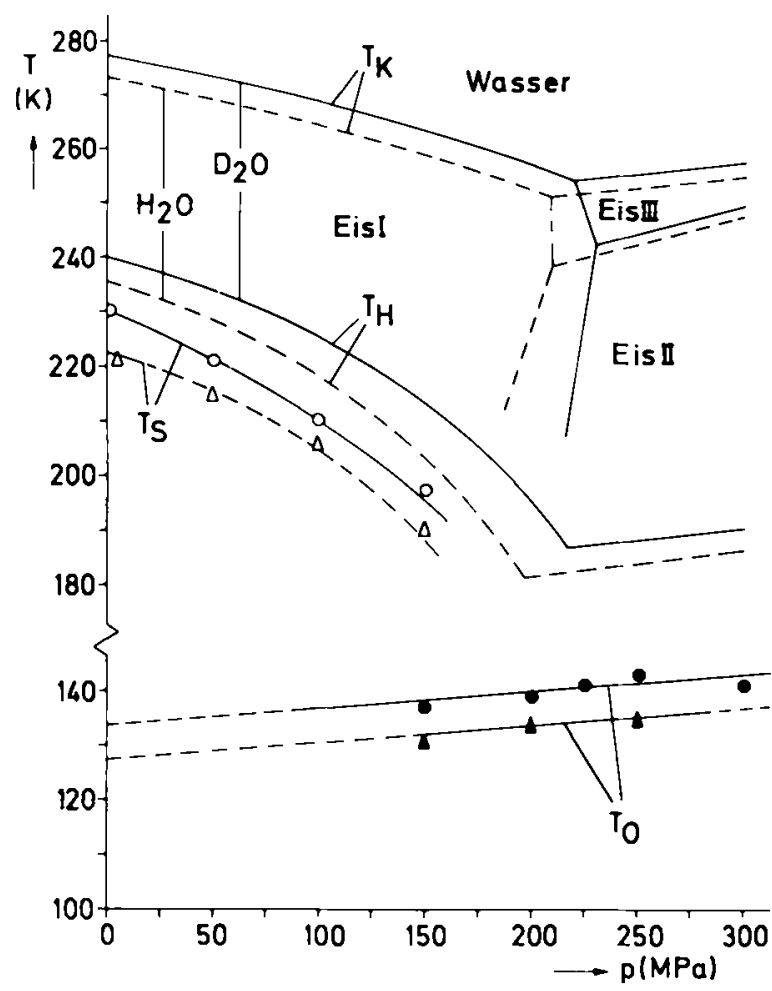

Fig. 12. p-T-Projektion des Phasendiagramms von $\mathrm{H}_{2} \mathrm{O}$ und $\mathrm{D}_{2} \mathrm{O}$, ergănzt. $T_{\mathrm{S}}$ Singularitátstemperatur, $T_{0}$ : ideale Glastemperatur. $\Delta_{1} \wedge: \mathrm{H}_{2} \mathrm{O}, 0, \bullet: \mathrm{D}_{2} \mathrm{O} . T_{\mathrm{S}}$ wurde aus der Anpassung der $\tau_{2}$-Daten an die Speedy-Angell-Gleichung gewonnen.

ten Werte sind ebenso wie die Hochdruckwerte in guter Übereinstimmung mit Literaturwerten der Glastemperatur $T_{0}{ }^{1176]}$. Ein Vergleich der im leichten und schweren Wasser gefundenen Werte läßt einen deutlichen Isotopeneffekt erkennen. In $\mathrm{D}_{2} \mathrm{O}$ ist $T_{0}$ um $\approx 6 \mathrm{~K}$ höher als in $\mathrm{H}_{2} \mathrm{O}$ (in Einklang mit einer im schweren Wasser geringeren Nullpunktsenergie der Moleküle). Auch alle anderen Parameter der VTF-Gleichung (10) zeigen einen Isotopeneffekt. Interessant ist die Isotopenabhängigkeit der Vorfaktoren $\tau_{0}$. Ihre Kehrwerte haben die Dimension einer Frequenz und entsprechen im Energiespektrum flüssigen Wassers den Librationsschwingungen der Moleküle, die dadurch zustandekommen, daß infolge der H-Brücken-Wechselwirkung ein

Tabelle 1. Parameter der VTF-Gleichung (10), die aus der Anpassung von $\tau_{2}$ an diese Gleichung erhalten wurden ( $\sigma=$ Standardabweichung).

\begin{tabular}{|c|c|c|c|c|c|c|c|c|}
\hline \multirow[t]{2}{*}{$p[\mathrm{MPa}]$} & \multicolumn{2}{|c|}{$T_{0} \pm 2[\mathrm{~K}]$} & \multicolumn{2}{|c|}{$B \pm \sigma[\mathrm{K}]$} & \multicolumn{2}{|c|}{$\left(\tau_{11} \pm \sigma\right) \cdot 10^{-14}[\mathrm{~s}]$} & \multicolumn{2}{|c|}{ Korr.-Koeff. } \\
\hline & $\mathrm{D}, \mathrm{O}$ & $\mathrm{H}, \mathrm{O}$ & $\mathrm{D}, \mathrm{O}$ & $\mathrm{H}_{2} \mathrm{O}$ & $\mathrm{D}, \mathrm{O}$ & $\mathrm{H}_{2} \mathrm{O}$ & $\mathrm{D}, \mathrm{O}$ & $\mathrm{H}_{2} \mathrm{O}$ \\
\hline 150 & 137 & 131 & $633 \pm 3$ & $652 \pm 5$ & $6.6 \pm 0.3$ & $4.6 \pm 0.3$ & 0.9998 & 0.9996 \\
\hline 200 & 139 & 134 & $604 \pm 3$ & $623 \pm 7$ & $6.1 \pm 0.5$ & $4.6 \pm 0.5$ & 0.9997 & 0.9992 \\
\hline 250 & 143 & 135 & $563 \pm 3$ & $581 \pm 5$ & $7.0 \pm 0.6$ & $5.9 \pm 0.3$ & 0.9997 & 0.9986 \\
\hline
\end{tabular}


Tabelle 2. Parameter der Speedy-Angell-Gleichung (12), die aus der Anpassung von $\tau_{2}$ an diese Gleichung erhalten wurden ( $\sigma=$ Standardabweichung).

\begin{tabular}{|c|c|c|c|c|c|c|c|c|}
\hline \multirow{2}{*}{$p[\mathrm{MPa}]$} & \multicolumn{2}{|c|}{$T_{\mathrm{b}} \pm 2[\mathrm{~K}]$} & \multicolumn{2}{|c|}{$\gamma \pm \sigma$} & \multicolumn{2}{|c|}{$\left(\tau_{\mathrm{S}} \pm \sigma\right) \cdot 10^{-t s}[\mathrm{~s}]$} & \multicolumn{2}{|c|}{ Korr.-Koeff. } \\
\hline & $\mathrm{D}, \mathrm{O}$ & $\mathrm{H}_{2} \mathrm{O}$ & $\mathrm{D}_{2} \mathrm{O}$ & $\mathrm{H}_{2} \mathrm{O}$ & $\mathrm{D}_{2} \mathrm{O}$ & $\mathrm{H}_{2} \mathrm{O}$ & $\mathrm{D}_{2} \mathrm{O}$ & $\mathrm{H}_{2} \mathrm{O}$ \\
\hline 0.1 & 230 & 223 & $1.85 \pm 0.01$ & $1.89 \pm 0.05$ & $3.74 \pm 0.1$ & $3.42 \pm 0.1$ & 0.9998 & 0.9991 \\
\hline 5 & 229 & 221 & $1.87 \pm 0.02$ & $1.87 \pm 0.02$ & $3.67 \pm 0.2$ & $3.67 \pm 0.2$ & 0.9993 & 0.9993 \\
\hline so & 221 & 215 & $1.93 \pm 0.02$ & $1.96 \pm 0.02$ & $4.23 \pm 0.1$ & $3.78 \pm 0.1$ & 0.9996 & 0.9997 \\
\hline 100 & 210 & 204 & $2.11 \pm 0.02$ & $2.11 \pm 0.02$ & $4.95 \pm 0.1$ & $4.95 \pm 0.1$ & 0.9994 & 0.9992 \\
\hline 150 & 196 & 189 & $2.37 \pm 0.1$ & $2.56 \pm 0.1$ & $6.73 \pm 0.2$ & $6.00 \pm 0.2$ & 0.9991 & 0.9978 \\
\hline
\end{tabular}

Molekül durch seine Nachbarn für eine gewisse Zeit in seiner Orientierung fixiert wird und um seine Gleichgewichtsorientierung oszilliert. In Einklang mit der Isotopenabhängigkeit der Librationsbanden ist das Verhältnis

$\frac{\tau_{0}^{-1}\left(\mathrm{D}_{2} \mathrm{O}\right)}{\tau_{0}^{-1}\left(\mathrm{H}_{2} \mathrm{O}\right)} \simeq \sqrt{\frac{I\left(\mathrm{D}_{2} \mathrm{O}\right)}{I\left(\mathrm{H}_{2} \mathrm{O}\right)}}=1.38$

gleich der Quadratwurzel aus dem Verhältnis der mittleren Trägheitsmomente der Moleküle. Sceats et al. ${ }^{[75]}$ interpretierten die Librationsbewegungen als thermische Anregungen im quasistatischen H-Brückennetzwerk. Demnach charakterisiert $\tau_{0}^{-1}$ die hochfrequenten Orientierungsfluktuationen der Moleküle in den Potentialmulden. Der Exponentialfaktor erfaßt den Einfluß der durch thermische Fluktuationen bewirkten topologischen Umordnung des ungeordneten $\mathrm{H}$-Brückennetzwerks. Wird dem System thermische Energie entzogen, so werden Konfigurationsfluktuationen immer seltener und damit die Orientierungskorrelationszeiten $\tau_{2}$ immer länger. Es ist zu erwarten, daß bei hohen Drücken auch die Temperaturabhängigkeit des Selbstdiffusionskoeffizienten und der Scherviskosität von diesen Fluktuationen bestimmt werden, also ebenfalls durch die VTF-Gleichung beschrieben werden. Für eine vollständige Überprüfung dieser Vermutung fehlen zur Zeit experimentelle Daten für tief unterkühltes Wasser bei hohen Drücken.

Bei niedrigeren Drücken $(p<150 \mathrm{MPa})$ wächst die Orientierungskorrelationszeit $\tau_{2}$ mit fallender Temperatur viel schneller ais es die VTF-Gleichung vorhersagt ${ }^{\text {[165, 166]. }}$ Wie die Diskussion der statischen Eigenschaften gezeigt hat, wird deren Temperaturabhängigkeit von einer thermodynamischen Singularität bei der Temperatur $T_{\mathrm{S}}$ bestimmt. Es soll deshalb untersucht werden, ob dies auch für dynamische Eigenschaften kalten flüssigen Wassers gilt. Die isobare Temperaturabhängigkeit der Orientierungskorrelationszeit $\tau_{2}$ bei niedrigen Drücken $(p \leq 150 \mathrm{MPa})$ wird quantitativ durch die von Speedy und $A$ ngell ${ }^{[93]}$ vorgeschlagene Gleichung (12), die aus der Theorie kritischer Erscheinungen bekannt ist, beschrieben:

$X=A_{\mathrm{x}}\left(\frac{T-T_{\mathrm{S}}}{T_{\mathrm{S}}}\right)^{-r_{\mathrm{x}}} \quad$ (Speedy-Angell-Gleichung)

Hierin ist $\gamma_{X}$ der kritische Exponent für die Eigenschaft $X$. Die mit Gl. (12) für leichtes und schweres Wasser erhaltenen Parameter sind in Tabelle 2 zusammengefaßt. Beim Übergang von $\mathrm{H}_{2} \mathrm{O}$ nach $\mathrm{D}_{2} \mathrm{O}$ ändert sich nur die Singularitätstemperatur $T_{\mathrm{S}}$. Fig. 13 zeigt, daß die bei beiden Flüssigkeiten für den gleichen Druck ermittelten Orientierungskorrelationszeiten $\tau_{2}$ bei Einführung der reduzierten Temperatur $T-T_{\mathrm{S}}$ in einem $\ln \tau_{2} \leftrightarrow \ln \left(T-T_{\mathrm{S}}\right)$-Diagramm auf einer Geraden liegen. Bei gleichem Abstand von der Sin-

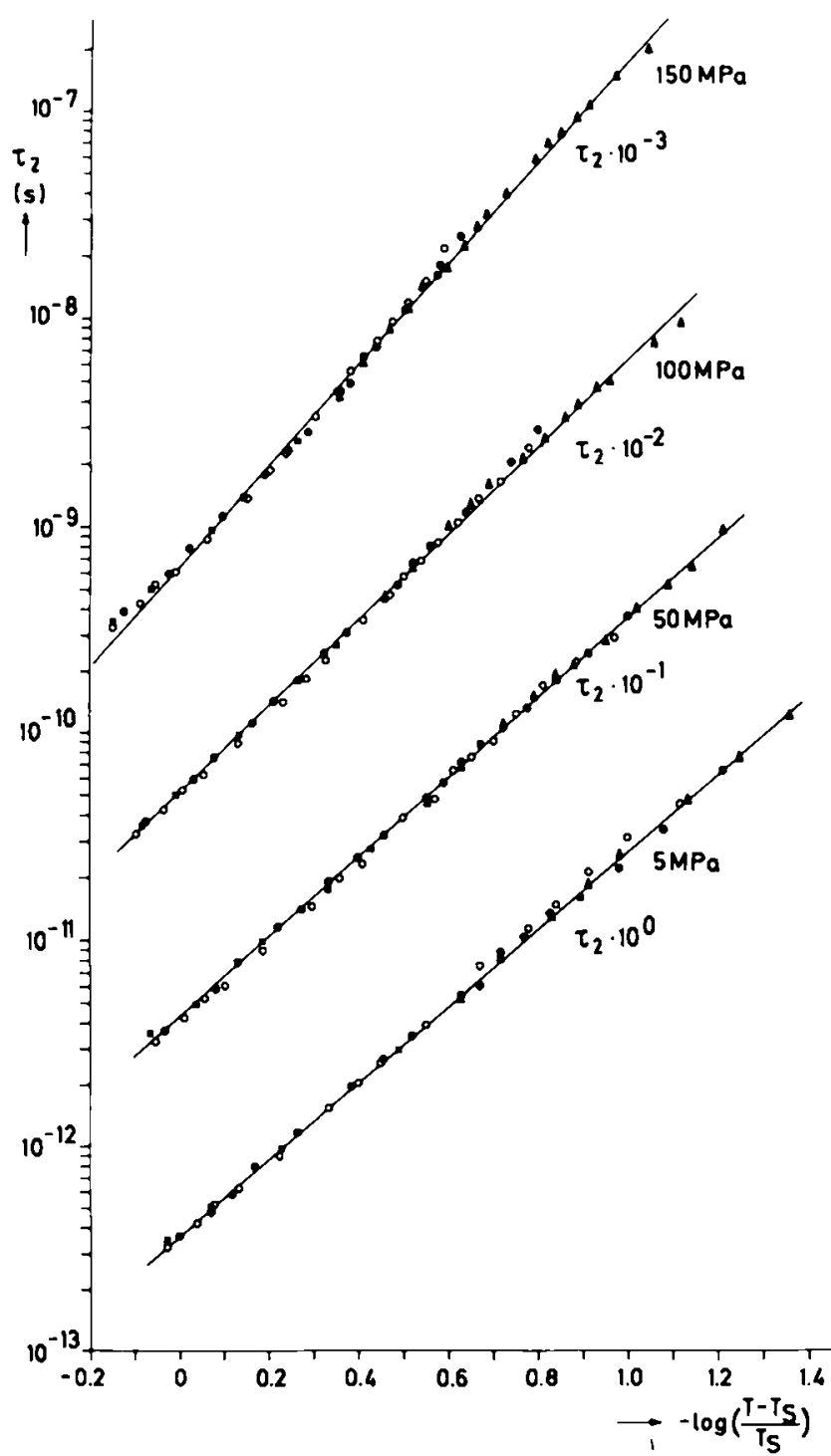

Fig. 13. Darstellung einiger $\tau_{2}$-Isobaren als Funktion der reduzierten Tempe raturen [166] ( $\tau_{2}$ : Orientierungskorrelationszeit, $T_{\mathrm{S}}$ : Singularitătstemperatur) o Berechnet aus ${ }^{17} \mathrm{O}-T_{1}$ in $\mathrm{H}_{2}{ }^{17} \mathrm{O}$; $\bullet$ berechnet aus ${ }^{17} \mathrm{O}-T_{1}$ in $\mathrm{D}_{2}{ }^{17} \mathrm{O} ; \Delta$ berechnet aus ' $H-T_{1}$ in $\mathrm{D}_{2} \mathrm{O}$ [165]; a berechnet aus ${ }^{2} \mathrm{H}-T_{1}$ in $\mathrm{D}_{2} \mathrm{O}[151,152]$.

gularitätstemperatur $\left(T-T_{\mathrm{S}}\right)$ ist das dynamische Verhalten der Wassermoleküle demnach gleich. Die den Faktoren $A_{\mathrm{X}} \equiv \tau_{\mathrm{S}}$ zugehörenden Frequenzen $\tau_{\mathrm{S}}^{-1}$ entsprechen im Spektrum des flüssigen Wassers den $\mathrm{O}-\mathrm{O}-\mathrm{O}$-Biegeschwingungen. Das Verhältnis der Frequenzen dieser behinderten Translationsschwingung ist bei einer Isotopensubstitution gleich der Wurzel aus dem Verhältnis der Molekülmassen $(=1.05)$. Es ist daher verständlich, daß innerhalb der Fehlergrenzen kein Isotopeneffekt für $\tau_{\mathrm{s}}$ gefunden wurde.

Die Ergebnisse legen es nahe, in den Fluktuationen der $\mathrm{H}$-Brückenbiegeschwingungen die Triebkraft für die anomalen Dichtefluktuationen zu suchen, deren zeitliches 
Anwachsen und Abklingen die Orientierungskorrelationsfunktion $G(t)$ bei niedrigen Drücken und tiefen Temperaturen kontrolliert.

Da die Orientierungskorrelationszeit durch

$\tau_{2}=J(\omega=0)=\int_{0}^{\infty} G(t) \mathrm{d} t$

definiert werden kann, folgt, daß die Orientierungsfluktuationen von den niederfrequenten Anregungen des unregelmäßigen Netzwerks dominiert werden.

$\mathrm{H}$-Brückenbiegeschwingungen treten um so deutlicher in Erscheinung, je geordneter und offener das unregelmäBige H-Brückennetzwerk ist. Sie sind also an das Vorhandensein geordneter Bereiche mit nahezu linearen H-Brükken gebunden. Wie die Diskussion der statischen Eigenschaften nahelegt, spiegeln die kooperativen OrdnungsUnordnungs-Fluktuationen im unregelmäßigen H-Brükkennetzwerk die Bildung solcher offenstrukturierter Bereiche bei Annäherung an $T_{\mathrm{S}}$ wider. Durch diese Prozesse wird auch die Umorientierung der Moleküle behindert, so daß die Orientierungskorrelationszeit $\tau_{2}$ mit Annäherung an $T_{\mathrm{S}}$ drastisch anwächst.

In diesem Zusammenhang interessiert auch, ob $D$ und $\eta$ dem gleichen Temperaturgesetz gehorchen. Die dynamischen Eigenschaften $D$ (Selbstdiffusionskoeffizient), $\eta$ (Scherviskosität) und $\tau_{2}$ (Orientierungskorrelationszeit) des flüssigen Wassers können bei Normaldruck durch die Stokes-Einstein-Gleichung (14) und die Debye-Gleichung (15) quantitativ miteinander verknüpft werden:

$D=k T / 4 \pi n a$

$\tau_{2}=4 \pi a^{3} \eta / 3 k T$

Messungen ergaben, daß die Beziehungen $\tau_{2} \sim \eta / T \sim 1 / D$ auch für die unterkühlte Phase gelten und $\mathrm{da} \beta$ die dynamischen Größen von $\mathrm{H}_{2} \mathrm{O}$ und $\mathrm{D}_{2} \mathrm{O}$ bei Einführung der reduzierten Temperaturen $\left(T-T_{\mathrm{S}}\right)$ auf einer Geraden liegen. Innerhalb der Fehlergrenzen haben alle Kurven die gleiche Steigung entsprechend $\gamma=-1.9 \pm 0.1$. Die plausible Erklärung hierfür ist, daß die Bildung geordneter Bereiche im unregelmäßigen $\mathrm{H}$-Brückennetzwerk sowohl die Umorientierung und Selbstdiffusion verlangsamt als auch die Scherfestigkeit des H-Brückennetzwerks erhöht.

\section{Unterkühlte wäßrige Salzlösungen}

Der Einfluß gelöster Stoffe auf Struktur und Dynamik des Wassers muß sich in unterkühlten Lösungen stärker als oberhalb der Schmelztemperatur bemerkbar machen. Fig. 14 faßt einige Messungen der Spin-Gitter-Relaxationszeit $T_{1}$ für Deuterium in unterkühlten Salzlösungen zusammen ${ }^{[176]}$. Der spezifische Einfluß der Ionen auf $T_{1}$ ist bei $238 \mathrm{~K}$ wesentlich ausgeprägter als bei $283 \mathrm{~K}$. Besonders interessant ist in diesem Zusammenhang, daß bei tiefen Temperaturen und niedrigen Drücken alle Ionen die Beweglichkeit der Wassermoleküle erhöhen. Unter diesen Bedingungen wirken also Magnesium- und Lithium-lonen als Strukturbrecher.

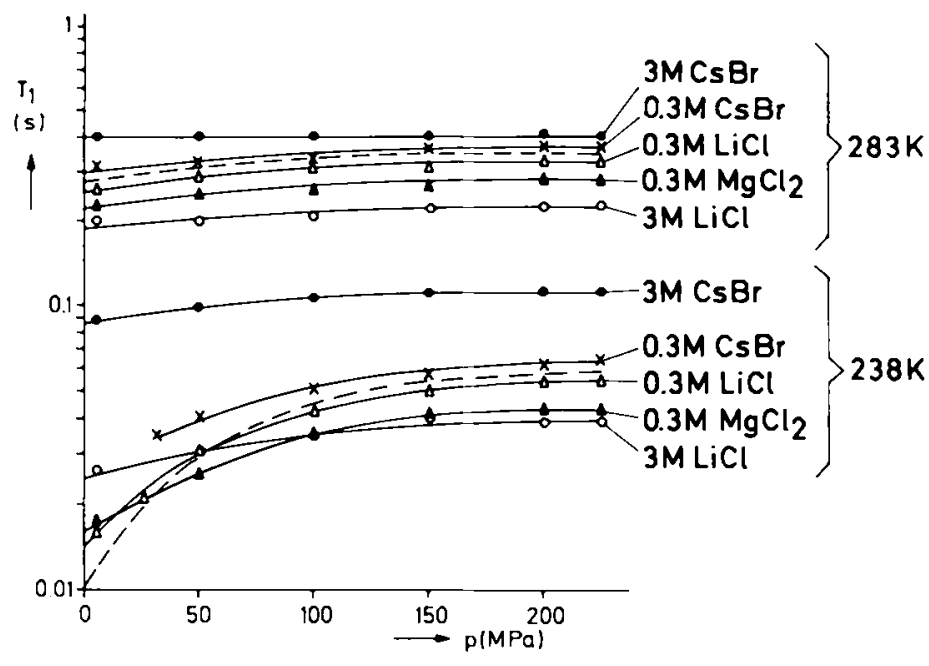

Fig. 14. Druckabhängigkeit von Isothermen der Deuterium-Spin-Gitter-Relaxationszeit $T_{1}$ einiger Salzlösungen in $\mathrm{D}_{2} \mathrm{O}$.

\section{Zusammenfassung und Ausblick}

Flüssiges Wasser muß als ein raumausfüllendes $\mathrm{H}$-Brükkennetzwerk aufgefaßt werden, in welchem lokal eine nahezu tetraedrische Anordnung nächster Nachbarn bevorzugt ist. Eine breite Verteilung von $\mathrm{O}-\mathrm{O}-\mathrm{O}$-Winkeln führt zu einem Verlust der Lagekorrelation für weiter entfernte Moleküle. Bei thermischer Anregung des Netzwerks kommt es zu behinderten Translationen und Librationen; strukturelle Veränderungen vollziehen sich auf der Zeitskala von Diffusionsprozessen. Der Entzug thermischer Energie stabilisiert lineare $\mathbf{H}$-Brücken und begünstigt Bereiche mit schlechter Raumausfüllung und somit geringer Dichte. Besonders im unterkühlten Wasser lagern sich diese Bereiche zusammen.

Als Folge dieser kooperativen Vorgänge sind viele thermodynamische und dynamische Größen im metastabilen Zustandsgebiet sehr stark temperaturabhängig. Offen bleibt die Frage, ob man sich mit fallender Temperatur einem Phasenübergang zweiter Ordnung, einer mechanischen Stabilitätsgrenze oder einer Perkolationsschwelle nähert. Hydrostatischer Druck destabilisiert die H-Brükkenbindungen und führt zu einer dichteren Packung der Moleküle im Netzwerk. Die Bildung geordneter Bereiche mit geringer Dichte wird zunehmend erschwert, die kooperativen Ordnungs-Unordnungs-Fluktuationen werden also mit steigendem Druck unterdrückt. Unter hohem hydrostatischen Druck zeigt Wasser eine Druck- und Temperaturabhängigkeit seiner physikalischen Eigenschaften, wie sie auch in vielen einfachen, viskosen Flüssigkeiten gefunden wird. Bis jetzt sind nur wenige Messungen physikalischer Eigenschaften im tief unterkühlten Wasser unter hohem Druck bekannt geworden. Die bei Experimenten im metastabilen Bereich zu überwindenden Hindernisse sind erheblich, insbesondere, wenn sich die Meßmethoden nicht auf die relativ beständigen Alkan/Wasser-Emulsionen anwenden lassen.

Computer-Simulationen sind für unterkühltes Wasser noch nicht durchgeführt worden, da das starke Anwachsen der mikroskopischen Zeitkonstanten bei Annäherung an $T_{\mathrm{H}}$, die Temperatur der homogenen Nucleation, den $\mathrm{Re}$ chenzeitbedarf enorm erhöht. Rechnungen im metastabilen Zustandsgebiet könnten jedoch zeigen, ob die gegen- 
wärtig verwendeten Potentialfunktionen imstande sind, das kooperative Verhalten der Moleküle zu beschreiben. Die im unterkühlten Wasser sehr viel stärker ausgeprägten Anomalien sind für alle Simulationen ein wichtiger Testfall.

Es ist zu erwarten, daß die experimentelle Untersuchung unterkühlter wäßriger Lösungen von Biopolymeren, apolaren Stoffen und Elektrolyten erheblich zum Verständnis dieser Lösungen im Stabilitätsbereich beitragen wird ${ }^{[17-181]}$ Die Analyse der dynamischen Eigenschaften des Wassers in unterkühlten Lösungen von Biopolymeren sollte, da man große Abweichungen vom Verhalten des reinen Wassers vermuten muß, zu neuen Einsichten in die Hydratation dieser Stoffe führen. Durch Messung der kinetischen Aktivität und der spektroskopischen Eigenschaften von Enzymen unter diesen Bedingungen läßt sich der untersuchbare dynamische Bereich enzymchemischer Reaktionen um Größenordnungen erweitern ${ }^{[195]}$, so daß instabile Reaktionszwischenstufen angereichert und spektroskopisch charakterisiert werden können ${ }^{[180,181]}$. Viele wechselwarme Lebewesen der Polarregionen produzieren während der kalten Jahreszeit „Antigefrier"-Stoffe, die meist zur Stoffklasse der Glycoproteine gehören ${ }^{[196-199]}$. Die Bildung dieser Stoffe ermöglicht es den Lebewesen, lange Zeit einige Grad unterhalb des „normalen“ Schmelzpunktes ihrer Körperflüssigkeit zu existieren. Der physikalische Mechanismus dieses Gefrierschutzes ist noch nicht eindeutig geklärt; es sollte aber gelingen, durch kernmagnetische Relaxation der Wassermoleküle in stark unterkühlten Lösungen einige der diskutierten Mechanismen kritisch zu überprüfen.

Weiterhin sollte es auch möglich werden, unterkühlte Emulsionen der Lösungen apolarer Stoffe in Wasser zu untersuchen und damit unsere Kenntnisse der hydrophoben Solvatation und hydrophoben Wechselwirkung zu vertiefen.

\section{Anhang}

Nach Fertigstellung dieses Fortschrittsberichtes veröffentlichten $W u$, Whalley und Dolling Hochdruckdaten bis $1560 \mathrm{MPa}$ zur Neutronenstreuung in $\mathrm{D}_{2} \mathrm{O}^{[200]}$. Es zeigt sich, daß die Zahl der nächsten Nachbarn von $0.1 \mathrm{MPa}$ bis zum höchsten erreichten Druck um ca. 50\% absteigt. Der erste $\mathrm{O}-\mathrm{O}$-Abstand bleibt dagegen praktisch unverändert. Außerdem wurde eine Arbeit von Angell, Sichina und Oguni ${ }^{[201]}$ angekündigt, die revidierte $C_{l}$-Werte für unterkühltes $\mathrm{H}_{2} \mathrm{O}$ und $\mathrm{D}_{2} \mathrm{O}$ bringt. Die grundsätzlichen Folgerungen von Abschnitt 3.3 werden durch diese Revision nicht berührt. Schließlich gelang es Speed ${ }^{[201]}$, das Verhalten des unterkühlten und überhitzten füssigen Wassers durch die Annahme einer geschlossenen mechanischen Stabilitätsgrenze für die Flüssigkeit zu beschreiben.

Wir danken Prof. C. A. Angell (West Lafayette, Ind. USA), Dr. A. Geiger (Aachen), Prof. R. J. Speedy (Wellington, Neuseeland) und Prof. H. E. Stanley (Boston, Mass., USA) für die Übersendung von Manuskripten vor der Veröffentlichung. Die eigenen Beiträge zu diesem Thema wurden durch großzügige Beihilfen der Deutschen Forschungsgemeinschaft und des Fonds der Chemischen Industrie gefördert.

Eingegangen am 30. November 1981 [A 410]
[1] M. Eigen, Naturwissenschaften 58 (1971) 465

[2] M. Eigen, Ber. Bunsenges. Phys. Chem. 80 (1976) 1059

[3] H. Kuhn, Ber. Bunsenges. Phys. Chem. 80 (1976) 1209.

[4] H. Kuhn, J. Waser, Angew. Chem. 93 (1981) 495; Angew. Chem. Int. Ed. Engl. 20 (1981) 500

[5] F. Franks: Water - A Comprehensive Treatise. Plenum Press, New York

[6] F. Franks in [5], Vol. 2 (1973), S. 1 ff.; F. Franks, D. S. Reid in [5], Vol. 2 (1973), S. $323 \mathrm{ff}$.

[7] H. Berendsen in [S], Vol. 5 (1975), S. $293 \mathrm{ff}$

[8] J. L. Finney in [5], Vol. 6 (1979), S. $47 \mathrm{ff}$.

[9] F. Franks in [S], Vol. 4 (1975), S. 1 ff.

[10] A. Ben-Naim: Hydrophobic Interactions, Plenum Press, New York 1980.

[11] S. C. Mossop, Proc. Phys. Soc. (London) B 68 (1955) 193

[12] V. J. Schaeffer, Ind. Eng. Chem. 44 (1952) 1300.

[13] D. H. Rasmussen, A. P. McKenzie in H. H. Jellinek: Water Structure and the Water-Polymer Interface. Plenum Press, New York 1972, S. $126 \mathrm{ff}$.

[14] D. H. Rasmussen, A. P. McKenzie, J. Chem. Phys. 59 (1973) 5003.

[15] H. Kanno, R. J. Speedy, C. A. Angell, Science 189 (1975) 880

[16] C. A. Angell, H. Kanno, Science 193 (1976) 1121

[17] J. P. Hansen, I. R. McDonald: Theory of Simple Liquids. Academic Press, London 1976.

[18] H. L. Lemberg. F. H. Stillinger, Mol. Phys. 32 (1976) 353.

[19] H. C. Andersen, J. Chem. Phys. 59 (1973) 4714.

[20] H. C. Andersen, J. Chem. Phys. 61 (1975) 4985.

[21] F. Vesely: Computerexperimente an Flüssigkeitsmodellen. Physik Verlag, Weinheim 1978.

[22] a) M. L. Klein, I. R. McDonald, B. J. Berne, M. Rao, B. L. Beveridge, P K. Mehrotra, J. Chem. Phys. $7 /$ (1979) 3889; b) M. Rao, C. Pangali, B. J. Berne, Mol. Phys. 37 (1979) 1773; c) M. Mezei, S. Swaminathan, D. L. Beveridge, J. Chem. Phys. 71 (1979) 3366.

[23] A. F. C. Ladd, Mol. Phys. 33 (1977) 1039

[24] a) W. K. Röntgen, Ann. Phys. (Leipzig) 45 (1892) 91; b) A. Eucken, Nachr. Ges. Wiss. Götringen 1946, 38.

[25] W. A. P. Luck, Angew. Chem. 92 (1980) 29: Angew. Chem. Int. Ed. Engl. 19 (1980) 28.

[26] D. Hankins, J. W. Moskowitz, F. H. Stillinger, J. Phys. Lett. 4 (1970) 527.

[27] B. R. Lentz, H. A. Scheraga, J. Chem. Phys. 58 (1973) 5296

[28] E. S. Campbell, M. Mezei, J. Chem. Phys. 67 (1977) 2338.

[29] F. H. Stillinger, Adv. Chem. Phys. 31 (1975) 1.

[30] A. Ben-Naim: Water and Aqueous Solutions, Plenum Press, New York 1974.

[31] D. W. Wood in [5], Vol. 6 (1979), S. $279 \mathrm{ff}$.

[32] P. Barnes in C. A. Croxton: Progress in Liquid Physics, Wiley, New York 1978, S. $391 \mathrm{ff}$

[33] F. H. Stillinger, J. Phys. Chem. 74 (1970) 3677.

[34] F. H. Stillinger, J. Chem. Phys. 57 (1972) 1780

[35] M. D. Morse, S. A. Rice, J. Chem. Phys. 74 (1981) 6514

[36] A. Ben-Naim, F. H. Stillinger in R. A. Horne: Structure and Transport Processes in Water and Aqueous Solutions. Wiley-Interscience, New York 1972.

[37] A. Rahman, F. H. Stillinger, J. Chem. Phys. 55 (1971) 3336.

[38] F. H. Stillinger, A. Rahman, J. Chem. Phys. 57 (1972) 1281.

[39] A. Rahman, F. H. Stillinger, J. Am. Chem. Soc. 95 (1973) 7943.

[40] F. H. Stillinger, A. Rahman, J. Chem. Phys. 60 (1974) 1545.

[41] F. H. Stillinger, A. Rahman in J. Lascombe: Molecular Motions in Liquids. D. Reidel, Dordrecht 1974, S. $479 \mathrm{ff}$

[42] A. Rahman, F. H. Stillinger, Phys. Rev. A 10 (1974) 368

[43] F. H. Stillinger, A. Rahman, J. Chem. Phys. 61 (1974) 4973.

[44] J. S. Rowlinson, Trans. Faraday Soc. 47 (1951) 120

[45] R. O. Watts, Mol. Phys. 28 (1974) 1069.

[46] R. O. Watts, Chem. Phys. 57 (1981) 185.

[47] W. L. Jorgensen, J. Am. Chem. Soc. 103 (1981) 335

[48] O. Matsuoka, E. Clementi, M. Yoshimine, J. Chem. Phys. 64 (1976) 1351

[49] G. C. Lie, E. Clementi, M. Yoshimine, J. Chem. Phys. 64 (1976) 2314

[50] D. C. Rapaport, H. A. Scheraga, Chem. Phys. Lelt. 78 (1981) 491.

[51] J. C. Owicki, H. A. Scheraga, J. Am. Chem. Soc. 99 (1977) 7403.

[52] F. T. Marchese, P. K. Mehrotra, D. L. Beveridge, J. Phys. Chem. 85 (1981) L 1 .

[53] W. L. Jorgensen, Chem. Phys, Lett. 70 (1980) 326

[54] H. L. Lemberg, F. H. Stillinger, J. Chem. Phys. 62 (1975) 1677.

[55] A. Rahman, F. H. Stillinger, H. L. Lemberg, J. Chem. Phys. 63 (1975) 5223 .

[56] F. H. Stillinger, A. Rahman, J. Chem. Phys. 68 (1978) 666

[57] R. O. Watts, Chem. Phys. 26 (1977) 367.

[58] I. R. McDonald, M. L. Klein, J. Chem. Phys. 68 (1978) 4875.

[59] I. R. McDonald, M. L. Klein, Faraday Discuss, Chem. Soc. 66 (1978) 48.

[60] F. H. Stillinger, C. W. David, J. Chem. Phys. 69 (1978) 1473

[61] C. W. David, Chem. Phys. 40 (1979) 229. 
[62] F. H. Stillinger, J. Chem. Phys. 71 (1979) 1647.

[63] F. H. Stillinger, C. W. David, J. Chem. Phys. 73 (1980) 3384.

[64] C. W. David, J. Chem. Phys. 73 (1980) 5395.

[65] C. W. David, Chem. Phys. Lell. 78 (1981) 488.

[66] C. W. David, Chem. Phys. Lett. 78 (1981) 337

[67] R. W. Impey, M. L. Klein, I. R. McDonald, J. Chem. Phys. 74 (1981) 647.

[68] S. Swaminathan, D. L. Beveridge, J. Am. Chem. Soc. 99 (1977) 8392.

169] M. Mezei, D. L. Beveridge, J. Chem. Phys. 74 (1981) 622.

[70] A. Geiger, F. H. Stillinger, A. Rahman, J. Chem. Phys. 70 (1979) 4185

[71] D. Eisenberg, W. Kauzmann: The Siructure and Properties of Water Oxford University Press, Oxford 1969.

[72] F. Hirata, P. J. Rossky, J. Chem. Phys. 74 (1981) 6867.

[73] A. C. Belch, S. A. Rice, M. G. Sceats, Chem. Phys. Lett. 77 (1981) 455

[74] S. A. Rice, M. G. Sceats, J. Phys. Chem. 85 (1981) 1108.

[75] M. G. Sceats, M. Stavola, S. A. Rice, J. Chem. Phys. 70 (1979) 3927.

[76] S. A. Rice, Top. Curr. Chem. 60 (1975) 109.

[77] M. G. Sceats, S. A. Rice, J. Chem. Phys. 72 (1980) 3236.

[78] M. G. Sceats, S. A. Rice, J. Chem. Phys. 72 (1980) 3248.

[79] M. G. Sceats, S. A. Rice, J. Chem. Phy's. 72 (1980) 3260.

[80] M. G. Sceats, S. A. Rice, J. Chem. Phys. 72 (1980) 6183.

[81] C. A. Angell in [5], Vol. 7, im Druck.

[82] B. V. Zheleznyi, Russ. J. Phys. Chem. 43 (1969) 1311.

[83] Handbook of Chemistry and Physics, S2. Aufl., CRC, Cleveland 1972, F 4 .

[84] W. S. Groenier, G. Thodos, J. Chem. Eng. Data 5 (1960) 285.

[85] M. Alei, Jr., W. M. Litchman, J. Chem. Phys. 57 (1972) 4106

[86] H. Kanno, C. A. Angell, J. Chem. Phys. 73 (1980) 1940

[87] G. Tammann, W. Jellinghaus, Z. Anorg. Allg. Chem. 174 (1928) 225

[88] E. M. Amagat, Ann. Chim. Phys. 29 (1893) 127.

[89] S. Lussana, Nuovo Cimento 2 (1895) 233

[90] S. J. Henderson, R. J. Speedy, J. Sci. Insirum. 13 (1980) 778.

[91] G. S. Kell, J. Chem. Eng. Dara 20 (1975) 97.

[92] G. S. Kell, J. Phys. Chem. Ref. Daia 6 (1977) 1109

[93] R. J. Speedy, C. A. Angell, J. Chem. Phys. 65 (1976) 851

[94] W. Seitz, G. Lechner, Ann. Phys. (Leipzig) 49 (1916) 93.

[95] R. E. Gibson, O. H. Loeffler, J. Am. Chem. Soc. 63 (1941) 898

[96] H. Kanno, C. A. Angell, J. Chem. Phys. 70 (1979) 4008

[97] G. S. Parks, J. Am. Chem. Soc. 47 (1925) 338.

[98] K. K. Kelley, J. Am. Chem. Soc. 5 I (1929) 779.

[99] G. S. Parks, H. M. Huffman, J. Am. Chem. Soc. 48 (1926) 2788.

[100] W. F. Giauque, J. W. Stout, J. Am. Chem. Soc. 58 (1936) 1144.

[101] Handbook of Chemistry and Physics, 52. Aufl., CRC, Cleveland 1972, D 128.

[102] E. A. Long, J. D. Kemp, J. Am. Chem. Soc. 58 (1936) 1829

[103] A. Eucken, M. Eigen, Z. Elektrochem. 55 (1951) 343.

[104] M. A. Anisimov, A. V. Voronel, N. S. Zaugolnikova, G. J. Ovodov, JETP Lelt. 15 (1972) 317.

[105] C. A. Angell, J. C. Tucker, Science 181 (1973) 342

[106] C. A. Angell, J. Shuppert, J. C. Tucker, J. Phys. Chem. 77 (1973) 3092

[107] C. A. Angell, J. C. Tucker, J. Phys. Chem. 84 (1980) 268.

[108] M. Oguni, C. A. Angell, J. Chem. Phys. 73 (1980) 1948.

[109] J. A. McMillan, S. C. Los, Nature 206 (1965) 806

[110] M. Sugisaki, H. Suga, S. Seki, Bull. Chem. Soc. Jpn. 41 (1968) 2591

[111] G. P. Johari, Philos. Mag. 35 (1977) 1077.

[112] E. Trinh, R. E. Apfel, J. Chem. Phys. 69 (1978) 4245.

[113] S. A. Rice, M. S. Bergren, L. Swingle, Chem. Phys. Lett. 59 (1978) 14.

[114] P. Brüggeller, E. Mayer, Nature 288 (1980) 569.

[115] J. B. Hasted, M. Shahidi, Nature 262 (1976) 777.

[116] J. M. Hodge, C. A. Angell, J. Chem. Phys. 68 (1978) 1363

[117] W. Dannhauser, L. W. Bake, J. Chem. Phys. 40 (1964) 3058.

[118] D. J. Denny, R. H. Cole, J. Chem. Phys. 23 (1955) 1767.

[119] G. Billaud, A. Demortier, J. Phys. Chem. 79 (1975) 3053.

[120] K. G. Breitschwerdt, W. Schmidt, Z. Naturforsch. A 25 (1970) 1467.

[121] M. Buback, W. D. Harder, Ber. Bunsenges. Phys. Chem. 81 (1977) 603

[122] M. Buback, W. D. Harder, Ber. Bunsenges. Phys. Chem. 81 (1977) 610.

[123] F. H. Stillinger, Philos. Trans. R. Soc. (London) B 278 (1977) 97.

[124] J. Rouch, C. C. Lai, S.-H. Chen, J. Chem. Phys. 66 (1977) 5031.

[125] J. Teixeira, J. Leblond, J. Phys. (Paris) Lett. 39 (1978) L-83.

[126] J.-C. Bacri, R. Rajaonarison, J. Phys. (Paris) Letr. 40 (1979) L-403.

[127] E. Trinh, R. E. Apfel, J. Chem. Phys. 72 (1980) 6731.

[128] M. Greenspan, C. E. Tschiegg, J. Acoust. Soc. Am. 31 (1959) 75

[129] G. D'Arrigo, Nuovo Cimento B 51 (1979) 304.

[130] H. E. Stanley, J. Phys. A 12 (1979) L 329.

[131] F. H. Stillinger in S. P. Rowland: Water in Polymers, American Chemical Society, Washington D.C. 1981, S. 11 If.

[132] H. E. Stanley, J. Teixeira, J. Chem. Phys. 73 (1980) 3404.

[133] H. E. Stanley, J. Teixeira, A. Geiger, R. L. Blumberg, Physica A 106 (1981) 260 .

[134] G. H. A. Cole in C. A. Croxton: Progress in Liquid Physics, Wiley, Chichester 1978, S. $1 \mathrm{ff}$.

[135] L. Bosio, J. Teixeira, H. A. Stanley, Phys. Rev. Lett. 46 (1981) 597.

[136] R. Zwanzig. Annu. Rev. Phys. Chem. 16 (1965) 67.

[137] W. F. van Gunsteren, H. J. C. Berendsen, J. A. C. Rullmann, Faraday Discuss. Chem. Soc. 66 (1978) 58.
[138] G. Williams, Chem. Soc. Rev. 7 (1978) 89.

[139] Shu-Yuan Chu, G. Sposito, J. Chem. Phys. 69 (1978) 2539.

[140] B. J. Berne, J.-P. Boon, S. A. Rice, J. Chem. Phys. 45 (1966) 1086

[141] G. Sposito, J. Chem. Phys. 74 (1981) 6943.

[142] A. B. Basset: A Treatise on Hydrodynamics. Dover, New York 1961.

[143] J. G. Powles, G. Rickayzen, Mol. Phys. 33 (1977) 1207.

[144] A. A. Maryott, M. S. Malmberg, K. T. Gillen, Chem. Phys. Lett. 25 (1974) 169.

[145] G. S. Kell in [5], Vol, 1 (1972), S. $404 \mathrm{ff}$.

[146] K. Tödheide in [5], Vol. 1 (1972), S. 482ff

[147] A. Harlow, Thesis, Imperial College London 1967.

[148] Ye. A. Osipov, B. V. Zheleznyi, N. F. Bondarenko, Russ. J. Phys. Chem. $5 l(1977) 1264$

[149] J. Hallett, Proc. R. Soc. 82 (1963) 1046

[150] N. A. Agayev in J. Straub, K. Scheffler: Water and Steam, Pergamon Press, Oxford 1980, S. $362 \mathrm{ff}$

[151] T. De Fries, J. Jonas, J. Chem. Phys. 66 (1977) 896.

[152] T. De Fries, J. Jonas, J. Chem. Phys. 66 (1977) 5393.

[153] K. T. Gillen, D. C. Douglass, M. J. R. Hoch, J. Chem. Phys. 57 (1972) 5117

[154] K. Krynicki, C. D. Green, D. W. Sawyer, Faraday Discuss. Chem. Soc 66 (1978) 199.

[155] C. A. Angell, E. D. Finch, L. A. Woolf, P. Bach, J. Chem. Phys. 65 (1976) 3063.

[156] D. J. Wilbur, T. De Fries, J. Jonas, J. Chem. Phys. 65 (1976) 1783.

[157] H. W. SpieB in P. Diehl, E. Fluck, R. Kosfeld: NMR - Basic Principles and Progress. Springer, Berlin 1978, Vol. 15, S. $55 \mathrm{ff}$.

[158] J. A. Glasel in [5], Vol. 1 (1972), S. $215 \mathrm{ff}$

[159] J. C. Hindman, J. Chem. Phys. 60 (1974) 4488.

[160] J. Jonas, Comments Solid Siate Phys. 8 (1977) 29.

[161] T. De Fries, J. Jonas, J. Chem. Phys. 66 (1977) 5393.

[162] T. De Fries, J. Jonas, J. Chem. Phys. 66 (1977) 896.

[163] W. J. Lamb, J. Jonas, J. Chem. Phys. 74 (1981) 913.

[164] E. W. Lang, H.-D. Lüdemann, J. Chem. Phvs. 67 (1977) 718.

[165] E. W. Lang, H.-D. Lüdemann, Ber. Bunsenges. Phys. Chem. 84 (1980) 462.

[166] E. W. Lang, H.-D. Lüdemann, Ber. Bunsenges. Phys. Chem. 85 (1981) 603.

[167] J. Hauer, E. W. Lang, H.-D. Lūdemann, Chem. Phys. 62 (1981) 195.

[168] H. G. Hertz, C. Rădle, Z. Phys. Chem. 68 (1969) 324.

[169] J. Hauer, E. W. Lang, H.-D. Lüdemann, Ber. Bunsenges. Phys. Chem. 83 (1979) 1262.

[170] J. Jonas, Ber. Bunsenges. Phys. Chem. 75 (1971) 257.

[171] J. Jonas, Annu. Rev. Phys. Chem. 26 (1975) 167.

[172] G. Harrison: The Dynamic Properties of Supercooled Liquids, Academic Press, London 1976

[173] H. Vogel, Phys. Z. 22 (1972) 645

[174] G. Tammann, W. Hesse, Z. Anorg. Allg. Chem. 156 (1926) 245.

[175] G. S. Fulcher, J. Am. Ceram. Soc. 77 (1925) 3701.

[176] E. W. Lang, Dissertation, Universităt Regensburg 1980

[177] A. P. MacKenzie, Philos. Trans. R. Soc. (London) B 278 (1977) 167

[178] D. H. Rasmussen, M. N. MacAulay, A. P. MacKenzie, Cryobiologie 12 (1975) 328.

[179] D. H. Rasmussen, A. P. Mackenzie, J. Phys. Chem. 75 (1971) 967

[180] P. Douzou, P. Debey, F. Franks, Biochim. Biophys. Acta 523 (1978) I.

[181] J. T. Edsall, H. A. McKenzie, Adu. Biophys. 10 (1978) 137.

[182] E. Kálmàn, G. Pálinkás, P. Kovács, Mol. Phys. 34 (1977) 505.

[183] E. Kálmán, G. Pálinkás, P. Kovács, Mol. Phys. 34 (1977) 525

[184] M. Alei, Jr., W. M. Litchman, J. Chem. Phys. 57 (1972) 4106.

[185] C. A. Hutchison, D. E. O'Reilly, J. Chem. Phys. 34 (1961) 163

[186] K. Krynicki, J. W. Hennel, Acta Phys. Pol. 24 (1963) 269.

[187] C. A. Hutchison, D. E. O'Reilly, J. Chem. Phys. 52 (1970) 4400.

[188] Landolt-Börnstein. Springer, Berlin 1969, Vol. 2, S. 208.

[189] D. E. O'Reilly, E. M. Peterson, C. E. Scheie, J. Chem. Phys. 58 (1973) 4072 .

[190] D. W. McCall, D. C. Douglas, E. W. Anderson, Phys. Fluids 4 (1961) 1317

[191] R. Haul, Th. Dorfmuller, Z. Naturforsch. A 19 (1964) 100

[192] K. C. Pratt, W. A. Wakeham, J. Chem. Soc. Faraday Trans. 73 (1977) 997.

[193] J. Jonas, T. De Fries, D. J. Wilbur, J. Chem. Phys. 65 (1976) 582.

[194] F. J. Dietz, J. J. de Groot, E. U. Franck, Ber. Bunsenges. Phys. Chem. 85 (1981) 1005 .

[195] P. Douzou, Adv. Enzymol. Relat. Areas Mol. Biol. $S I$ (1980) 1.

[196] J. G. Duman, J. L. Patterson, J. J. Kozak, A. L. DeVries, Biochim. Biophys. Acta 626 (1980) 332.

[197] D. Slaughter, G. L. Fletcher, V. S. Ananthanarayanan, C. L. Hew, J. Biol. Chem. 256 (1981) 2022

[198] A. I. Ahmed, D. T. Osuga, R. E. Feeney, Biochem. Int. I (1980) 41

[199] J. A. Raymond, A. L. DeVries, Proc. Natl. Acad. Sci. USA 74 (1977) 2589.

[200] A. Y. Wu, E. Whalley, G. Dolling, Chem. Phys. Lett. 84 (1981) 433.

[201] C. A. Angell, W. J. Sichina, M. Oguni, J. Phys. Chem. 86 (1982), im Druck.

[202] R. J. Speedy, J. Phys. Chem. 86 (1982), im Druck. 